\title{
Tabularia
}

\section{Un pouvoir d'abbé en acte(s) : Raoul d'Argences, abbé de Fécamp (1190-1219)}

The power of an abbot via act(s): Raoul of Argences, abbot of Fécamp (1190-1219)

\section{Fabien Paquet}

\section{OpenEdition}

Journals

Édition électronique

URL : http://journals.openedition.org/tabularia/1293

DOI : 10.4000/tabularia.1293

ISSN : 1630-7364

Éditeur :

CRAHAM - Centre Michel de Boüard, Presses universitaires de Caen

Référence électronique

Fabien Paquet, "Un pouvoir d'abbé en acte(s) : Raoul d'Argences, abbé de Fécamp (1190-1219) », Tabularia [En ligne], Actes épiscopaux et abbatiaux en Normandie et dans le grand Ouest européen, mis en ligne le 13 décembre 2011, consulté le 30 avril 2019. URL : http://journals.openedition.org/ tabularia/1293 ; DOI : 10.4000/tabularia.1293 


\title{
Un pouvoir d'abbé en acte(s): Raoul d'Argences, abbé de Fécamp (1190-1219) ${ }^{1}$
}

\section{The power of an abbot via act(s): Raoul of Argences, abbot of Fécamp (1190-1219)}

\author{
Fabien PAQUET \\ ENS de Lyon \\ fabien.paquet@ens-lyon.fr
}

Résumé:

Après la conquête de 1204, tous les abbés bénédictins de Normandie deviennent des abbés royaux; Philippe Auguste renforce ainsi les liens qui existaient depuis le XII e siècle entre le pouvoir capétien et les prélats normands. Ce moment représente un tournant dans l'histoire de tous les monastères de la principauté. L'étude de l'abbatiat de Raoul d'Argences à la Trinité de Fécamp (1190-1219) contribue à mettre en lumière la politique des abbés autour de 1204. À travers l'examen des actes de cet abbé, cet article entend montrer que moins qu'un temps d'instabilité, le changement d'autorité royale a été un moment de renforcement du pouvoir abbatial à plusieurs titres, pour ce qui est de l'abbaye de Fécamp. L'étude des aspects diplomatiques et celle des objets de quarante-cinq chartes de Raoul concordent, et montrent que les abbés doivent aussi être considérés sous leur volet politique.

Mots-clés: abbayes, abbés, diplomatique abbatiale, Fécamp, Normandie, Philippe II Auguste, Raoul d'Argences, XIII ${ }^{\mathrm{e}}$ siècle.

\section{Abstract:}

As soon as Normandy was attached to the Royal domain, Philip II Augustus gave Royal status to all the Norman Benedictine abbots and reinforced links that existed since the $X I I^{\text {th }}$ century between the Capetian rulers and the Norman prelats. This moment represents a turn in the history of all the monasteries of the principality. The study of Raoul of Argences, abbot of la Trinité of Fécamp (1190-1219) has brought new light on the politics of Abbots around 1204. Following the examination of acts of this particular abbot, this article aims to show that far from being a moment of instability, the change of Royal power was a moment when abbatial authority was, in as far as the Abbey of Fécamp is concerned, reinforced. The study of the diplomatic aspects and the subject of forty-five charters of Raoul concord, and show that the political position of abbots must also be taken into consideration.

Keywords: abbeys, abbots, abbatial diplomacy, Fécamp, Normandy, Philip II Augustus, Raoul of Argences, XIII ${ }^{\text {th }}$ century.

1. Je tiens à remercier Sébastien Barret, Michaël Bloche, Véronique Gazeau et Sylvain Gouguenheim pour leurs conseils et relectures attentives.

Tabularia «Études», $\mathrm{n}^{\circ} 11,2011$, p. 49-79, 13 décembre 2011 http://www.unicaen.fr/mrsh/craham/revue/tabularia/print.php?dossier=dossier11\&file=02paquet.xml 
Quand Philippe Auguste annexe la Normandie au domaine royal capétien en 1204, une de ses premières mesures est d'attacher les abbayes à sa couronne, en rendant royaux tous les abbés bénédictins normands. Pour l'abbaye de Fécamp, cette mesure a une portée particulière, du fait des liens entretenus avec le pouvoir angevin jusqu'au XII e siècle. L'abbatiat de Raoul d'Argences représente à ce titre un tournant dans l'histoire de l'abbaye bénédictine de la Trinité de Fécamp². Vingt-neuf ans à la tête d'un des premiers monastères de Normandie, il gère le passage de la domination Plantagenêt à la domination capétienne avec lucidité, veillant au maintien, voire à l'extension des privilèges de son monastère. Son abbatiat ouvre alors un XIII siècle fécampois marqué par un essor de l'abbaye à plusieurs points de vue (territorial, financier, réputation, etc.) ${ }^{3}$.

Originaire d'Argences, près des grands domaines bas-normands de l'abbaye, Raoul d'Argences, moine de Fécamp ${ }^{5}$, est élevé sur le trône abbatial en 1190. Plutôt proche du pouvoir Plantagenêt dans les années $1190^{6}$, Raoul sait ménager le pouvoir capétien après 1204, se ralliant fidèlement à Philippe Auguste et obtenant de lui la confirmation, et l'extension de ses privilèges. En 1211, le droit de haute justice lui est concédé par le roi de $\mathrm{France}^{7}$; il s'ajoute à l'exemption de l'abbaye, que Raoul avait fait confirmer par Célestin III par plusieurs bulles entre 1193 et $1197^{8}$. A la tête d'un domaine s'étendant des deux côtés de la Manche, qui comprend notamment des droits importants sur plusieurs grands ports de la Manche (Fécamp, Le Tréport, Rye, Winchelsea), Raoul réussit à maintenir et à accroître ce domaine après $1204^{9}$. Au détour de l'étude d'une affaire concernant le village de Boissy-Mauvoisin ${ }^{10}$, Léopold Delisle avait déjà remarqué l'importance de cet abbatiat pour la Trinité de Fécamp ${ }^{11}$.

Nous avons la chance de disposer d'un important corpus de documents pour l'étudier: nous avons jusque-là identifié quarante-cinq chartes de Raoul, dont

2. Dép. Seine-Maritime, chef-lieu de cant.

3. C'est ce qui ressort des recherches que nous menons sous la direction de Véronique Gazeau sur les abbés normands du XIII ${ }^{e}$ siècle.

4. Argences: dép. Calvados, cant. Troarn. Au sujet des terres bas-normandes de l'abbaye, voir Six, 2002, $\mathrm{n}^{\circ}$ 2, p. 99-127 et $\mathrm{n}^{\circ} 3$, p. 225-251.

5. Chronique des abbés de Fécamp, ou "Histoire de l'abbaye de Fécam par je ne sçais quel auteur... », Leroux De LinCy, Antoine (éd.), in Leroux De LinCy, 1840, p. 293.

6. Il est ainsi fermier de la vicomté de Fécamp à au moins deux reprises (1194-1195 et 1202-1203: Stapleton, Magni Rotuli..., vol. 1, p. 168 et 560; Hardy, Rotuli Litterarum Patentium..., p. 30). Pendant la guerre entre Jean sans Terre et Philippe Auguste, il reçoit une lettre confidentielle du roi angevin, que l'archidiacre de Wells (Somerset) doit rapporter au roi s'il ne peut la remettre à son destinataire (HARDY, Rotuli Litterarum Patentium...., p. 42 ; cette lettre est faite en quatre exemplaires. L'archidiacre de Wells s'en est vu confier deux par Jean : une à destination de Raoul et une autre pour le seigneur de Norwich (Norfolk)). Gazeau, 2007, vol. 2, p. 123.

7. Delisle, Cartulaire normand..., n ${ }^{\circ} 216$, p. $34 ; 217$ p. 34 et 299 et 725, p. 161.

8. RAmackers, Papsturkunden..., $\mathrm{n}^{\circ} 310,316,338$ et 339 .

9. Cela passa notamment par plusieurs voyages en Angleterre (HARDY, Rotuli Litterarum Patentium..., p. 56, 73 et 106) ainsi que par le choix comme prieur de Cogges (Oxfordshire), de Michael (ou Michel) d'Argences, son neveu (Arch. dép. Seine-Maritime, 7 H 24).

10. Dép. Yvelines, cant. Bonnières-sur-Seine.

11. Delisle, 1904. 
beaucoup ne nous sont parvenues que sous la forme de copie dans deux cartulaires (XIII ${ }^{\mathrm{e}}$-XIV ${ }^{\mathrm{e}}$ siècle) de l'abbaye ${ }^{12}$ : nous ne disposons que de neuf actes originaux ${ }^{13}$. Ce corpus n'a guère été étudié jusqu'à présent; seule la charte de Raoul confirmant l'octroi du droit de justice par Philippe Auguste a été éditée, par Léopold Delisle ${ }^{14}$. Cette étude ne prétend pas en proposer une exploitation exhaustive. Deux temps - la diplomatique des actes et leurs objets - viseront à montrer que l'abbatiat de Raoul représente un moment de codification et de stabilisation pour l'abbaye de Fécamp, dans un contexte qui n'y semble pas propice. Il s'agit d'approfondir l'étude d'un abbatiat qui a donné à la Trinité de Fécamp les bases d'un «beau XIII siècle», ce qui permettra, à l'échelle d'une abbaye, d'éclairer une petite partie de la transition Plantagenêt-Capétien pour la Normandie, un élément de ce que Lucien Musset et avant lui Charles Petit-Dutaillis, ont nommé la «naissance d'une province ${ }^{15}$.

\section{La stabilisation d'une diplomatique}

Les travaux concernant la diplomatique abbatiale sont peu nombreux: même Alfred Giry dans son imposant Manuel de diplomatique n'y consacre que de rares paragraphes ${ }^{16}$. Nous manquons donc très souvent de moyens de comparaison. Cette étude des actes de Raoul d'Argences espère lancer quelques pistes qui seront utiles à l'étude d'autres corpus ${ }^{17}$.

\section{L'émetteur des actes: l'abbé}

Comment l'abbé figure-t-il dans les actes? Michaël Bloche propose dans ce dossier une étude de la suscription des actes des abbés de Fécamp du XI ${ }^{\mathrm{e}}$ siècle

12. Cf. annexe.

13. Annexe, acte $\mathrm{n}^{\circ}$ I, III, VII, X, XI, XIII, XIV, XVI et XLIV. Nous avons écarté les notices qui ne mentionnent qu'indirectement un acte de Raoul d'Argences.

14. Delisle, Cartulaire normand..., n $\mathrm{n}^{\circ} 217$ p. 34 et 299.

15. Petit-Dutaillis, 1925, p. 99. Musset, 1982, p. 291. Cet aspect de l'histoire de la Normandie (la conquête et le XIII ${ }^{\mathrm{e}}$ siècle) est le parent pauvre de l'histoire normande depuis la mort de Léopold Delisle qui n'a pas pu tirer tous les renseignements de l'imposant corpus de sources qu'il a éditées. M. Powicke a tout de même proposé une étude solide de la chute des Plantagenêt en Normandie (Powicke, 1913). Les travaux de D. Power ont permis d'avoir une vision bien plus juste encore de cette période (voir en particulier Power, 2004). D. Power est également un des rares historiens de la Normandie à consacrer des études au XIII ${ }^{\mathrm{e}}$ siècle, en témoigne par exemple son article, "CrossChannel communication and the end of the 'Anglo-Norman realm': Robert fitzWalter and the Valognes inheritance», Tabularia, «Études», $\mathrm{n}^{\circ}$ 11, 2011, p. 1-33. Le cœur du XIII siècle, malgré quelques travaux sporadiques (comme STRAYER, Joseph R., The administration of Normandy under Saint Louis, Cambridge, The mediaeval Academy of America, 1932, réimpr. 1970), reste fort peu connu.

16. GIRY, 1925.

17. Quelques études de corpus limités dans le temps et/ou l'espace existent cependant, comme: GASPARRI, Françoise, "Observations sur les chartes originales de l'abbaye Saint-Victor de Paris au XII" siècle», Scrittura e civiltà, t. 23, 1999, p. 157-175; CHAPLAIs, Pierre, "The Original Charters of Herbert and Gervase, Abbots of Westminster (1121-1157)", in BARnES, Patricia et SLAdE, Cecil Frederick (eds), A Medieval Miscellany for Doris Mary Stenton, Londres, The Pipe Roll Society, 1962, p. 89-110. 
au début du XIVe siècle; nous renvoyons à son article à ce propos ${ }^{18}$. Retenons, ainsi qu'il le montre, une stabilisation de la formule de suscription sous Raoul, apparaissant souvent ainsi: Sciant presentes et futuri quod ego Radulfus, Dei gratia humilis abbas Fiscannensis, et totus ejusdem loci conventus... Celle-ci n'est cependant pas figée. On rencontre ainsi à trois reprises cette autre formule: Universis Sancte Matris ecclesie filiis presens scriptum inspecturis, Radulfus, miseratione divina humilis abbas Fiscannensi, totusque ejusdem loci conventus salutem in Domino ${ }^{19}$. La nature des actes n'explique pas les changements.

L'abbé de Fécamp est l'émetteur unique de tous les actes sauf deux: les societates conclues en 1215 avec Saint-Denis en France et Saint-Lucien de Beauvais ${ }^{20}$, dont les textes débutent ainsi: Notum sit omnibus, tam presentibus quam futuris, quod inter conventum Beati Dyonisii et conventum Fiscannensi, tempore venerabili Henrici Beati Dyonisii et Radulphi de Argenciis Fiscannensi abbatum hujusmodi constituta est societas ${ }^{21}$. Pour une autre charte, cependant, Raoul est l'auteur de l'acte, écrit et juridique, mais n'est pas à l'initiative de l'action concernée:

Sciant presentes et futuri quod ego Radulfus, Dei gratia humilis abbas Fiscannensi, et totus ejusdem loci conventus, ad petitionem karissimi domini nostri Philippi Dei gratia illustris regis Francorum, concessimus Ricardo Syme, servienti suo et fideli amico nostro, quandam vavassoriam apud Heudebouvilla ${ }^{22}$, que fuit Gaufridus de Orgevallis, salvo omni jure tam nostro quam alieno, cum omnibus illis que ad predictam vavassoriam pertinent. Teste universitate capituli nostri. Testibus etiam Ricardo de Passorio, Willelmo de Duno et aliis ${ }^{23}$.

Établi après 1204, ce document reflète le passage de l'abbé de Fécamp sous souveraineté capétienne. Les deux partis tirent avantage de cette façon d'émettre l'acte: Raoul d'Argences reste l'émetteur officiel - Philippe Auguste n'empiète donc pas sur ses prérogatives - tandis que le pouvoir du capétien s'affirme sur un de ses nouveaux hommes et sur ses nouvelles terres, puisque les biens concernés font bien partie de l'extension du domaine royal de 1204 . La désignation du vavasseur concerné va en ce sens: serviteur du roi, il est aussi l'«ami» de l'abbé de Fécamp, la portée de ce terme restant cependant difficile à juger.

Toujours associé à l'abbé dans la suscription, le chapitre occupait sous Raoul d'Argences un rôle non négligeable dans les actes abbatiaux.

18. Notamment pour ce qui est de l'usage du qualificatif «humilis» et de l'association du chapitre à l'abbé dans la suscription.

19. Arch. dép. Calvados, H 4368. Bibl. mun. Rouen, ms 1207 [Y 51], fol. 32V, 33 et 38-v.

20. Dép. Oise, chef-lieu de cant.

21. Arch. dép. Seine-Maritime, $7 \mathrm{H} 51$.

22. Dép. Eure, cant. Louviers.

23. Bibl. mun. Rouen, ms 1207 [Y 51], fol. 34 . 


\section{Autour de l'abbé: les témoins}

Sous Raoul d'Argences, se généralise la pratique de prendre à témoin l'ensemble du chapitre de l'abbaye. C'est le cas dans vingt des quarante-cinq actes étudiés. La formule Teste universitate capituli nostri figure alors dans l'eschatocole, le plus souvent entre la mention du sceau et la liste des témoins. Elle peut être associée ou non à d'autres témoins, alors introduits par la formule Testibus etiam ${ }^{24}$. La plupart du temps, cependant, elle est employée seule (seize fois sur vingt occurrences). Ce moyen de validation, fort courant, que l'on ne rencontre guère avant l'abbatiat de Raoul, correspond au moment, ainsi que le note M. Bloche, à partir duquel le couvent est associé à l'abbé dans la suscription de presque tous les actes ${ }^{25}$. On peut considérer que le recours au témoignage de tout le chapitre est une réponse logique à son association dans l'autorité émettrice de l'acte: en quelque sorte, le chapitre est à la fois auteur et témoin. Cela correspond à la gestion des terres du monastère par Raoul d'Argences. Elle passait par la nomination de proches - ce qui relevait parfois du népotisme - à la tête des principaux offices de l'abbaye: son neveu, Achard, est ainsi écolâtre ${ }^{26}$, et nombre de personnages sont dits de Argenciis sans que l'on connaisse leurs liens précis avec l'abbé. Cette formule est promise à un certain avenir à Fécamp: sous les abbés Guillaume Vaspail (1227-126o ${ }^{27}$, Richard de Treigos (126o-1284/1286) ${ }^{28}$, puis au XIV ${ }^{\mathrm{e}}$ siècle dans sa version française: «tesmoing l'université de notre capitre». On retrouve la même expression dans les actes d'autres abbayes, toujours au XIII ${ }^{\mathrm{e}}$ siècle, mais semble-t-il moins systématiquement que dans les actes de Raoul: ainsi en 1205, dans un acte de l'abbé de Marmoutier ${ }^{29}$ ou en 1244 dans une charte de l'abbé de Sainte-Catherine de Rouen ${ }^{30}$.

La généralisation de cette formule suggère une homogénéisation des listes de témoins sous Raoul d'Argences: l'étude des témoins, bien que ceux-ci soient fort nombreux, va dans ce sens également. Sur les quarante-cinq actes étudiés, vingt-trois en comprennent une liste. Leur étude est soumise à caution car la plupart sont connus à partir de leur copie dans le cartulaire de l'abbaye, qui ne reprend pas les listes complètes des témoins: ainsi, un vidimus d'une charte d'Henri de Sully, prédécesseur de Raoul, est souscrit par au moins huit personnes dans sa version originale ${ }^{31}$, tandis que sa copie dans le cartulaire ne reprend que le premier, "et multis aliis» ${ }^{32}$. Cette réduction de la liste des

24. Des chartes où seul le chapitre est témoin : Bibl. mun. Rouen, ms 1207 [Y 51], fol. 31v, 32; 33v. Des chartes où le chapitre est associé à d'autres témoins: ibid., fol. 34, 35; 37.

25. Article en préparation, pour ce dossier de Tabularia.

26. Arch. dép. Seine-Maritime, 7 H 9, p. 6.

27. Bibl. mun. Rouen, ms 1207 [Y 51], fol. 35.

28. Arch. dép. Seine-Maritime, $7 \mathrm{H} 909$.

29. Marmoutier: dép. Indre-et-Loire, c. Tours (chef-lieu de cant.). GesLin de Bourgogne et BARTHÉLÉMY, 1864, nº XIII, p. 228.

30. Delisle, Cartulaire normand..., $\mathrm{n}^{\circ} 452$, p. 74.

31. Arch. dép. Seine-Maritime, $7 \mathrm{H} \mathrm{24}$; la charte est endommagée, rendant difficile la lecture de la liste de témoins.

32. Bibl. mun. Rouen, ms 1207 [Y 51], fol. 34v, 35. 
témoins est plus nette dans le cartulaire conservée à la bibliothèque municipale de Rouen ${ }^{33}$ que dans celui conservé aux Archives de la Seine-Maritime ${ }^{34}$. L'étude des listes n'est néanmoins pas impossible, dans la mesure où nous disposons tout de même de quelques actes originaux, et où les témoins repris dans la liste du cartulaire sont les premiers de la liste, ceux donc qui sont a priori les plus importants.

Soixante-dix-sept témoins différents ont été relevés dans les vingt-trois chartes qui en comprennent une liste. Le nombre de témoins par charte était parfois assez important ${ }^{35}$. Une concession de biens à Rye ${ }^{36}$ est souscrite par quatorze personnes nommées et aliis multis ${ }^{37}$. Une autre concession de biens comprend dix témoins et multis aliis ${ }^{38}$. La confirmation par Raoul d'une donation à l'archidiacre de Fécamp par un bourgeois de la ville, Raoul Gernet, en comprend vingt-deux et multis aliis ${ }^{39}$. La confirmation d'une autre donation, dont Raoul est le garant, est également souscrite par vingt-deux témoins et multis aliis, répartis en deux listes ${ }^{40}$. La première liste (quinze témoins), qui clôt ce qui pourrait être une charte intégrale, est en effet suivie d'une nouvelle clause, elle-même confirmée par une seconde liste (dix témoins), dont certains noms figurent déjà dans la première liste, mais pas tous ${ }^{41}$. La première liste est introduite par l'habituel testibus, tandis que la seconde l'est par astantibus, seule occurrence de ce terme dans notre corpus.

Qui sont les témoins? Certains n'apparaissent qu'à une seule reprise, quand eux, leur famille ou leur seigneur sont concernés par le texte. En revanche, les hommes du parti de l'abbé reviennent assez régulièrement dans les listes, nous permettant ainsi de mettre en évidence le premier cercle du pouvoir de Raoul d'Argences. Les souscriptions d'officiers sont plus rares que sous ses prédécesseurs: c'est une conséquence directe du recours à la souscription de l'ensemble du chapitre. Quelques officiers figurent toutefois parmi les témoins de ses chartes: ainsi Hugues, aumônier, en souscrit-il cinq ${ }^{42}$ et Achard, cellérier,

33. Bibl. mun. Rouen, ms 1207 [Y 51].

34. Arch. dép. Seine-Martime, $7 \mathrm{H}$ 9. La formule « et multis aliis» (ou ses variantes) figure cependant aussi dans des chartes originales (par ex.: Arch. dép. Seine-Maritime, $7 \mathrm{H} \mathrm{43)}$; on ne doit donc pas toujours la comprendre comme une abréviation de la part des scribes qui ont copié ces cartulaires.

35. Le nombre de témoins cependant n'est pas le nombre des personnes ayant assisté à la transaction couchée dans l'acte.

36. Sussex.

37. Arch. dép. Seine-Maritime, $7 \mathrm{H} 43$.

38. Ibid., $7 \mathrm{H} 27$.

39. Ibid., $7 \mathrm{H}$ 9, p. 5 .

40. Ibid., 7 H 623. Copie du XIV siècle: Arch. dép. Seine-Maritime, 7 H 9, p. 5-6.

41. Les actes à plusieurs listes de témoins apparaissent au XI ${ }^{\mathrm{e}}$ siècle quand les actions juridiques deviennent plus complexes et que l'exécution des clauses recourt à plusieurs autorités (Tock, 2005, p. 230-234). La deuxième liste de notre acte suit une clause qui ajoute un personnage dans la transaction, le frère de Guillaume Moine, le donateur.

42. Arch. dép. Seine-Maritime, 7 H 9, p. 5; 7 H 43; 7 H 623. Bibl. mun. Rouen, ms 1207 [Y 51], fol. $60 v$. 
quatre ${ }^{43}$. Trois prieurs de prieurés sont témoins de chartes: Robert Arsic, prieur de Saint-Georges de Mantes (deux chartes) ${ }^{44}$, Michel d'Argences, prieur de Cogges (une charte) ${ }^{45}$, Julien, prieur de Saint-Gabriel (une charte) ${ }^{46}$. D'autres personnages prennent le pas sous Raoul d'Argences, qui font partie de ce qu'on peut nommer l'hôtel de l'abbé: en premier lieu le sénéchal et le chambrier.

Le sénéchal apparaît comme le vrai bras droit de l'abbé pour la gestion du temporel. Deux sénéchaux sont connus pour l'abbatiat de Raoul, Richard de Gislarville (ou de Gillarville) ${ }^{47}$ et Roger Panchevout. Richard de Gislarville est très présent dans les documents des années 1190-1210: il souscrit dix des quarante-cinq actes de Raoul ${ }^{48}$, ainsi qu'un très grand nombre d'actes concernant l'abbaye ${ }^{49}$. Il est difficile de dater précisément son sénéchalat: il est en poste en 1198, 1205, 1207 et 1215. Son successeur, Roger Panchevout, est en poste dès $1217^{50}$. On ignore si Richard de Gislarville était déjà en poste sous Henri de Sully, le prédécesseur de Raoul d'Argences: cela est possible, puisqu'on a la trace de sénéchaux qui restent en poste après la mort de l'abbé qui les a nommés ${ }^{51}$. Richard de Gislarville est très peu connu. Un «maittre Guillaume de Gislarville» souscrit une charte de 1204 aux côtés de Richard de Gislarville ${ }^{52}$. Un Guillaume de Gislarville (ou de Guillerville) est connu en qualité de théologien au XIII siècle; originaire de la même paroisse, celui-ci serait passé par Fécamp: pourrait-il s'agit du même personnage? Nous l'ignorons ${ }^{53}$. Le successeur de Richard, Roger Panchevout, est un peu mieux connu. Il est probablement originaire de Biville-la-Martel ${ }^{54}$. Il fait une donation à l'abbaye; on apprend par la même occasion qu'il dispose d'un sceau personnel ${ }^{55}$. Sa femme se prénomme

43. Arch. dép. Seine-Maritime, 7 H 9, p. 5; 7 H 24; 7 H 623. Bibl. mun. Rouen, ms 1207 [Y 51], fol. $60 \mathrm{v}$.

44. Bibl. mun. Rouen, ms 1207 [Y 51], fol. 35-v et 36-v. Saint-Georges de Mantes: dép. Yvelines, c. Mantes-la-Jolie, chef-lieu de cant.

45. Arch. dép. Seine-Maritime, 7 H 24. Cogges: Oxfordshire.

46. Bibl. mun. Rouen, ms 1207 [Y 51], fol. 36. Saint-Gabriel: dép. Calvados, cant. Creully, c. SaintGabriel Brécy.

47. Il s'agit très probablement de Guillerville, ancienne paroisse aujourd'hui rattachée à Bolleville (dép. Seine-Maritime, cant. Bolbec), connue sous les noms de Gillarville et Guillarvilla au XIII siècle: BeAUREPAIRE, 1979, p. 90.

48. Bibl. mun. Rouen, ms 1207 [Y 51], fol. 35-v., 37 et 6ov. Arch. dép. Seine-Maritime, 7 H 9, p. 5 ; $7 \mathrm{H} 27 ; 7 \mathrm{H} 623$

49. Par ex. : Arch. dép. Seine-Maritime, 7 H 9, p. 4, 6 et 8; Bibl. mun. Rouen, ms 1207 [Y 51], fol. 38bisv, 39 bis, $48,60-v, 68,77-v, 78 v, 81 v, 82,83-v, 84-v, 85$.

50. Arch. dép. Seine-Maritime, $7 \mathrm{H}$ 9, p. 2.

51. C'est le cas de Roger Panchevout, qui est encore en poste sous Aichard d'Argences, abbé de 1220 à 1222 et sous Richard Morin, abbé de 1222 à 1227 : Arch. dép. Seine-Maritime, 7 H 9, p. 2, 3, 4 et 36; Bibl. mun. Rouen, ms 1207 [Y 51], fol. 69 v, 72, 82v, 83, 90v et 91.

52. Arch. dép. Seine-Maritime, 7 H 9, p. 8. Le même Guillaume souscrit une autre charte, probablement plus tard: Bibl. mun. Rouen, ms 1207 [Y 51], fol. 91.

53. RosnY, 1809, p. 249. Histoire littéraire de la France, t. 32, Paris, 1898, p. 466-469 et 607.

54. Dép. Seine-Maritime, cant. Valmont, c. Ypreville-Biville. Bibl. mun. Rouen, ms 1207 [Y 51], fol. 91. L'abbaye de Fécamp possédait des biens à Biville-la-Martel (MaBILLE, 1953, p. 16).

55. Bibl. mun. Rouen, ms 1207 [Y 51], ms 1207, fol. 91. 
Alice ${ }^{56}$. On connaît assez mal les fonctions exactes du sénéchal. Richard de Gislarville est par exemple chargé de payer cent sous au nom de l'abbé à un Guillaume de Cuverville ${ }^{57}$ : Testibus Ricardo de Gislarvilla, tunc senescallo abbatis, per cui manu recepi prefatos centum solidos andegavensium de abbate et conventu Fiscannensi ${ }^{58}$. Il n'est donc pas étonnant qu'il soit aussi présent dans les listes de témoins. Nous n'avons en revanche aucune trace de charte émise par un de ces hommes en tant que sénéchal de l'abbé: il n'avait pas ce niveau d'autonomie vis-à-vis du pouvoir abbatial.

Le chambrier est l'autre officier récurrent. Sous Raoul d'Argences, il se nomme Guillaume et figure ainsi dans les listes: Willelmo clerico tunc domini abbatis camerario. Il souscrit quatre chartes de Raoul ${ }^{59}$, ainsi qu'un grand nombre d'autres chartes concernant l'abbaye ${ }^{60}$. Il souscrit la plupart du temps juste après le sénéchal. Le chambrier est un officier de l'abbaye normalement recruté parmi les moines: or, il arrive que sa souscription s'ajoute à celle de l'ensemble du chapitre, ce qui n'est jamais le cas pour les autres officiers: Teste universitate capituli nostri. Testibus etiam Ricardo de Gislarville, Willelmo clerico tunc camerario nostro et aliis. ${ }^{61}$. Faut-il en déduire que la souscription du chambrier, possible responsable du chartrier et des archives de l'abbaye ${ }^{62}$, avait une valeur propre, non diluable dans la souscription collective du chapitre? Un autre personnage, qui était très probablement moine de l'abbaye, avait une souscription particulière: le scribe des actes. Sous Raoul, nous avons la trace d'un scribe, Richard de Passeor ou de Passorio. Il figure ainsi dans la liste des témoins: Ricardo de Passorio, hujus scripti auctore ${ }^{63}$. Il arrive qu'il souscrive sans que soit mentionné le fait qu'il est l'auteur de la charte ${ }^{64}$; on peut néanmoins penser qu'il l'est, d'autant plus que ces mentions ont été rencontrées dans le cartulaire de la bibliothèque municipale de Rouen, dont le scribe a tendance à fortement abréger les listes de témoins. On trouve aussi sa souscription dans plusieurs chartes non émises par Raoul mais qui concernent Fécamp; il n'en est jamais mentionné comme l'auteur ${ }^{65}$. On ne sait presque rien de ce Richard, mis à part qu'il est chapelain et qu'il a un frère, Roger ${ }^{66}$.

56. Bibl. mun. Rouen, ms 1207 [Y 51], ms 1207, fol. 91

57. Dép. Calvados, cant. Troarn.

58. Bibl. mun. Rouen, ms 1207 [Y 51], ms 1207, fol. 84v.

59. Ibid., fol. $35 \mathrm{v}, 37$ et $60 \mathrm{v}$.

60. Ibid., fol. $60 \mathrm{v}, 79 \mathrm{v}, 84-\mathrm{v}$.

61. Ibid., fol. 37 .

62. Le chambrier étant en charge de la gestion des revenus de l'abbaye, conservés dans la chambre, où devaient aussi se trouver les archives de l'abbaye, on peut supposer qu'il gérait les documents concernant le temporel de l'abbaye (dont le cartulaire), tandis que le bibliothécaire devait gérer les fonds liturgiques et littéraires. Sur les fonctions du chambrier: SCHMITZ, 1942-1956, t. I, p. 282.

63. Arch. dép. Seine-Maritime, $7 \mathrm{H}$ 9, p. 5 et $7 \mathrm{H} \mathrm{623.} \mathrm{Cependant,} \mathrm{des} \mathrm{études} \mathrm{d'autres} \mathrm{corpus} \mathrm{ont}$ montré que le verbe scribere pouvait désigner tant «l'acte concret et matériel d'écrire», que «la responsabilité générale de l'écriture d'un acte» (Tock, 2005, p. 114; voir aussi ATsmA et VeziN, 2000, p. 15).

64. Bibl. mun. Rouen, ms 1207 [Y 51], fol. 34

65. Ibid., fol. $79 \mathrm{v}, 82,85,91 \mathrm{v}$.

66. Bibl. mun. Rouen, ms 1207 [Y 51], fol. 79v. Arch. dép. Seine-Maritime, 7 H 9, p. 7. 
Nombre de laïcs figurent aussi parmi les témoins des actes de Raoul, mais pour les étudier, il faut aussi prendre en compte leur présence dans les autres chartes de l'abbaye. Comme l'a remarqué Dominique Rouet dans son étude du cartulaire de Saint-Pierre de Préaux, certains vassaux de l'abbaye deviennent des "témoins professionnels", associés à un grand nombre d'actes ${ }^{67}$. Il est même probable qu'ils aient assisté à davantage de transactions que celles pour lesquelles nous avons leur souscription ${ }^{68}$. Le premier groupe est constitué des personnages dits de Argenciis. Leur nombre trahit une pratique népotiste. On rencontre ainsi Richard d'Argences ${ }^{69}$, Roger d'Argences ${ }^{70}$, Hubert d'Argences ${ }^{71}$, Arnoulf d'Argences ${ }^{72}$, Pérégrin d'Argences ${ }^{73}$, Guillaume d'Argences ${ }^{74}$, ainsi qu'un Nicolas, nepos de l'abbé ${ }^{75}$. Toutes ces occurrences ne sont pas dans les actes de Raoul d'Argences, mais témoignent de l'importance d'un des réseaux sur lesquels il appuie son pouvoir. D'autres familles sont récurrentes: Renaud et Roger de Brueria souscrivent un nombre important d'actes ${ }^{76}$; tout comme Guillaume, Thomas et Robert de Dune ${ }^{77}$, ou encore Richard, Robert et Guillaume de Camera $^{78}$. Avec toutes les précautions habituelles quant à l'étude des listes de témoins, on peut tout de même souligner la politique de Raoul qui consiste à associer à son pouvoir, parfois en récompensant ${ }^{79}$, quelques groupes de vassaux, en particulier pour la gestion des terres lointaines comme les domaines bas-normands de l'abbaye qui sont sa principale source de revenus normande ${ }^{80}$.

\section{La datation des actes}

$\mathrm{Au} \mathrm{XII}{ }^{\mathrm{e}}$ siècle, la datation des actes se généralise, dans un contexte de réappropriation du droit romain ${ }^{81}$ : qu'en est-il de ceux de Raoul d'Argences? Quatorze chartes de notre corpus présentent une date: date chronique pour toutes et date

67. Rouet, Le cartulaire..., p. LXXXVII.

68. TOCK, 2005, p. 229.

69. Bibl. mun. Rouen, ms 1207 [Y 51], fol. 39bisv, 55v, 62-v. et 77v. Arch. dép. Calvados, H 4368.

70. Ibid., fol. 39 bisv.

71. Ibid., fol. 77 .

72. Arch. dép. Seine-Maritime, 7 H 9, p. 4

73. Ibid., p. 4 et 5 .

74. Bibl. mun. Rouen, ms 1207 , fol. 39 bis, $59 \mathrm{v}, 60,76 \mathrm{v}, 83 \mathrm{v}$ et 85 .

75. Arch. dép. Seine-Maritime, $7 \mathrm{H} 43$.

76. Ibid., $7 \mathrm{H} \mathrm{9,} \mathrm{p.} \mathrm{1,} \mathrm{2,} \mathrm{4,} \mathrm{6,} \mathrm{16,} \mathrm{22;} 7 \mathrm{H} 43 ; 7 \mathrm{H} 623$.

77. Ibid., 7 H 9, p. 2, 6, 14, $22 ; 7$ H 43. Bibl. mun. Rouen, ms 1207 [Y 51], fol. 34.

78. Arch. dép. Seine-Maritime, 7 H 9, p. 2, 3 (?), 4, 5, 22; 7 H 623.

79. Par exemple: Bibl. mun. Rouen, ms 1207 [Y 51], fol. 32-v.: Raoul d'Argences concède à Robert Camerario (probablement un membre de la famille de Camera évoquée plus haut; les deux formes de leur nom se rencontrent régulièrement), un ministère ayant appartenu à un Guillaume, pour le service que ledit Robert a accompli dans la boulangerie de l'abbaye.

80. Au sujet des terres bas-normandes de l'abbaye, voir Six, 2002.

81. Guyotjeannin et alii, 1993, p. 84. La réappropriation du droit romain est moins forte en Normandie où la coutume garde une importance première - notamment sous l'impulsion capétienne - mais les effets de cette réappropriation sur l'ensemble des diplomatiques, en premier lieu celle du roi de France, et donc des barons du royaume, sont réels dans la principauté. 
topique pour trois. C'est assez peu (31\%) pour des actes du début du XIII siècle pour la plupart. Cela n'est cependant pas étonnant dans le contexte normand: le XII siècle est marqué dans l'espace angevin par l'absence de datation systématique des actes, ainsi que l'a par exemple noté Léopold Delisle pour les actes de Henri II ${ }^{82}$. A la même époque, les actes du prédécesseur de Raoul, Henri de Sully (1140-1187) comprennent très rarement une date ${ }^{83}$. Ajoutons que les chartes d'abbés sont connues pour leur absence de datation jusqu'aux XII ${ }^{e}$-XIII ${ }^{e}$ siècles ${ }^{84}$. C'est sous Raoul d'Argences que la mention de la date semble se généraliser dans les actes des abbés de Fécamp: après lui, elle est quasi-systématique. Même si on ne dispose que de peu de documents pour l'abbatiat de Guillaume Vaspail (1227-1260), ceux que nous connaissons sont tous datés ${ }^{85}$. Par ailleurs, les actes de Raoul qui sont datés sont pour onze d'entre eux de 1209 ou après. Un seul est daté du XII siècle: Hoc autem fuit [apud] Fiscannum in pleno capitulo, anno domini $M^{\circ} \mathrm{C}^{\circ} \mathrm{XC}^{\circ}$ sexto $^{86}$. Un est daté de 1201: Actum publice in capitulo, anno Verbi incarnati $\mathrm{M}^{\circ} \mathrm{CC}^{\circ}$ Primo $^{87}$. Peut-on alors en déduire que la mention de la date dans les actes est liée à la conquête de 1204 ? Peut-être que le contact avec la diplomatique franque, qui comprenait très régulièrement la date dès le $\mathrm{XII}^{e}$ siècle, a accéléré cette évolution pour les actes de Raoul. Dominique Rouet propose une même interprétation pour ce qui est de Saint-Pierre de Préaux, mais il faudrait mener une étude d'ensemble des actes des abbés normands de la transition, pour vérifier si une évolution semblable est constatable, et si la date apparaît en même temps dans toute la Normandie ${ }^{88}$. Les actes de Raoul d'Argences montrent en tout cas une stabilisation de la mention de la date à partir de 1209/1210. La date topique, quant à elle, disparaît, alors qu'elle était plus courante que la date chronique sous son prédécesseur, Henri de Sully ${ }^{89}$. Deux formules s'affirment pour la date chronique: Actum anno Gratie... (cinq occurrences $)^{90}$ et Actum anno Domini... (six occurrences) ${ }^{91}$. La première se rencontre en 1205 (une fois), en 1210 (une fois), 1211 (deux fois) et 1216 (une fois); mais c'est la seconde qui prend le pas sur la première à partir de 1213, et pour la suite du XIII e siècle, puisqu'on la retrouve dans les cinq actes connus de

82. Delisle (éd.), Recueil des actes..., p. 9-11. D. Rouet le montre également pour l'abbaye bénédictine de Saint-Pierre de Préaux (Rouet, Le cartulaire..., p. CII-CIII).

83. Exemples d'actes d'Henri non datés: Bibl. mun. Rouen, ms 1207 [Y 51], fol. 74v, 75; Arch. dép. Seine-Maritime, $7 \mathrm{H} \mathrm{24}, 7 \mathrm{H} 43$ et $7 \mathrm{H} 57$; BnF, ms lat. 564, fol. 1v. Au sujet d'Henri de Sully, voir GAZEAU, 2007, vol. 2, p. 116-121.

84. GuyotjeAnNin et alii, 1993, p. 84-85.

85. Bibl. mun. Rouen, ms 1207 [Y 51], fol. 39-v, 3obisv, 31bis et 35bis. Arch. dép. Seine-Maritime, $7 \mathrm{H} 27$.

86. Arch. dép. Seine-Maritime, $7 \mathrm{H} 27$.

87. Bibl. mun. Rouen, ms 1207 [Y 51], fol. 31v.

88. Rouet, Le cartulaire..., p. CII-CIII.

89. Par exemple: Arch. nat. S 4889 B: Actum est apud Fiscannum...; Arch. dép. Seine-Maritime, $7 \mathrm{H} 43$ : Actum publice apud Fiscannum...; $7 \mathrm{H} 2142$ : Data apud Fiscannum..

90. Bibl. mun. Rouen, ms 1207 [Y 51], fol. 32-v, 33 et 39; Delisle, Cartulaire normand..., n ${ }^{\circ}$ 216, p. 34 et 299. Teulet, Layettes..., t. I, $\mathrm{n}^{\circ} 744$, p. 281.

91. Bibl. mun. Rouen, ms 1207 [Y 51], fol. 31, 38-v. et 63-v. Arch. dép. Seine-Maritime, 7 H 51. Arch. dép. Calvados, H 4368. 
Guillaume Vaspail ${ }^{92}$. La date ne comprend souvent que l'année, mais le mois est mentionné à cinq reprises, soit dans le tiers de actes ${ }^{93}$; et dans deux autres cas, une précision est donnée: Actum anno Gratie $M^{\circ} C C^{\circ}$ decimo, die jovis in capite jeiunii ${ }^{94}$ et Actum anno domini $M^{\circ} C C^{\circ}$ quintodecimo in crastino dedicationis ecclesie nostre ${ }^{95}$ : dans les deux cas, il s'agit de la pratique habituelle et ancienne de datation à partir d'un évènement du calendrier de l'abbaye ${ }^{96}$. La mention du mois, assez rare sous Raoul d'Argences, devient systématique ensuite: tous les actes de Guillaume Vaspail la comprennent, ou, à défaut, la mention d'un événement du calendrier liturgique aisément datable ${ }^{97}$. Parallèlement à la régularisation de la mention de la date sous Raoul, l'emplacement de celle-ci dans l'eschatocole se fixe également: en 1196, on trouve la date puis les témoins ${ }^{98}$; en 1201, l'annonce du sceau puis la date ${ }^{99}$; en 1205 , les témoins puis la date ${ }^{100}$, en 1209, l'annonce du sceau puis la date puis les témoins ${ }^{101}$, en 1210, la date puis l'annonce du sceau, puis les témoins ${ }^{102}$; en 1211, la date puis les témoins ${ }^{103}$ et, à partir de 1213, systématiquement l'annonce du sceau, la date, puis les témoins ${ }^{104}$, ordre que l'on retrouve sous Guillaume Vaspail ensuite ${ }^{105}$.

Un acte nous permet enfin de proposer une hypothèse quant au style utilisé à Fécamp, en raison de sa date: Actum est autem hoc anno Gratie millesimo ducentesimo quarto, mense januario, apud Fiscannum ${ }^{106}$. Or, cet acte fait suite à un acte du prévôt de Boissy ${ }^{107}$, daté de novembre $1204^{108}$. Philippe Auguste y est en outre mentionné de telle façon qu'on comprend que la Normandie est passée sous domination capétienne: domino nostro Philippo, illustri Francorum regi. On peut donc en déduire que le style appliqué n'est ni celui de la Circoncision ( $1^{\mathrm{er}}$ janvier) ni celui de Noël, bien que celui-ci soit assez répandu dans l'espace angevin $^{109}$. La Normandie cependant s'inscrivait, dès le XII ${ }^{\mathrm{e}}$ siècle, dans le système français davantage que dans le système anglais: on peut donc penser

92. Bibl. mun. Rouen, ms 1207 [Y 51], fol. 39-v.; 3obisv; 31bis et 35bis. Arch. dép. Seine-Maritime, $7 \mathrm{H} 27$.

93. Teulet, Layettes..., t. I, $\mathrm{n}^{\circ}$ 744, p. 281. Bibl. mun. Rouen, ms 1207 [Y 51], fol. 63-v. Arch. dép. Seine-Maritime, $7 \mathrm{H} 51$ et $7 \mathrm{H} 2095$.

94. Bibl. mun. Rouen, ms 1207 [Y 51], fol. 32.

95. Ibid., fol. 38-v.

96. GIRY, 1925, p. 579-581.

97. Par exemple: die ante purificationem Beate Viginis [2 février] (Arch. dép. Seine-Maritime, $7 \mathrm{H} \mathrm{27).}$

98. Arch. dép. Seine-Maritime, $7 \mathrm{H} 27$.

99. Bibl. mun. Rouen, ms 1207 [Y 51], fol. 31v.

100. Teulet, Layettes..., t. I, $\mathrm{n}^{\circ}$ 744, p. 281.

101. Bibl. mun. Rouen, ms 1207 [Y 51], fol. 63-v.

102. Ibid., fol. 32 .

103. Ibid., fol. 39

104. Ibid. fol. 31, 38-v., etc.

105. Bibl. mun. Rouen, ms 1207 [Y 51], fol. 39-v.; 3obisv; 31bis et 35bis. Arch. dép. Seine-Maritime, $7 \mathrm{H} 27$.

106. Teulet, Layettes..., t. I, $\mathrm{n}^{\circ} 744$, p. 281.

107. Dép. Yvelines, cant. Bonnières-sur-Seine, c. Boissy-Mauvoisin.

108. Delisle, 1904, p. 391-392.

109. GIRY, 1925, p. 115 . 
que les actes de Raoul d'Argences étaient datés selon le style de Pâques ${ }^{110}$. Le fait qu'un acte de 1204/1205 soit déjà daté selon le style de Pâques tendrait à nous pousser à relativiser la radicalité du changement évoqué par Alfred Giry, Olivier Guyotjeannin et Benoît-Michel Tock pour la Normandie, selon le modèle "Noël jusqu'en 1204, Pâques ensuite " ${ }^{111}$. Ne peut-on pas penser que le style de Pâques avait déjà franchi la frontière de la principauté auparavant, du fait de l'augmentation des contacts entre l'Ile-de-France capétienne et les abbayes de Normandie, en particulier sous Louis VII ${ }^{112}$ ? Une étude prenant en compte un plus grand nombre d'abbayes sera indispensable pour répondre à cette question.

Considérant alors qu'à Fécamp, c'est probablement le style de Pâques qui domine à partir de l'abbatiat de Raoul d'Argences, on peut préciser la date de certains actes ${ }^{113}$. Un acte est daté du premier jour du Carême de 1210. La Pâques 1211 tombant le 3 avril, il est daté du 19 février 1211 (n. st. $)^{114}$. Ajoutons que Raoul ne date jamais ses actes en fonction des années de son abbatiat.

L'abbatiat de Raoul d'Argences semble donc représenter une étape centrale dans la fixation d'une diplomatique abbatiale. On peut rapprocher cela des remarques de David Chadd concernant la liturgie fécampoise: l'ordinaire qu'il édite date de l'abbatiat de Raoul, plutôt dans sa partie XIII ${ }^{\mathrm{e}}$ siècle ${ }^{115}$. Mais surtout, "unlike some examples of medieval liturgical ordinals, the Fécamp book shows evidence of having been planned and executed with attention to his appearance and long-term utility ${ }^{116}$. L'auteur inscrit bien la rédaction de ce manuscrit dans un contexte de réorganisation plus générale: reconstruction de l'église abbatiale, réorganisation des autels, réactivation de la légende du «Précieux Sang ${ }^{117}$. Cette démarche de normalisation se traduit-elle aussi dans l'objet des actes? Dans un temps touché par la guerre puis la conquête, peut-on, à travers ce corpus, déterminer quelles furent les priorités de Raoul d'Argences?

\section{Gérer un monastère normand pendant la transition Plantagenêt-Capétiens: typologie des actes de Raoul d'Argences}

Quelles sont les actions de Raoul d'Argences que transcrivent ses actes? Il est possible de dresser une typologie des actes d'un abbé d'une époque charnière.

110. Le style de Pâques a été adopté par le pouvoir capétien au XII ${ }^{e}$ siècle, probablement sous Louis VII (Guyotjeannin et Tock, 1999, p. 53). À Saint-Pierre de Préaux, D. Rouet montre que le style de Pâques remplace le style de Noël à partir de 1204 (Rouet, Le cartulaire..., p. CIV).

111. Guyotjeannin et Tock, 1999, p. 70; en partie d'après Giry, 1925, p. 115.

112. Arch. dép. Seine-Maritime, $9 \mathrm{H} 24$ (arbitrage de Louis VII en faveur de l'abbaye de Jumièges); Luchaire (éd.), Étude sur les actes de Louis VII, n² 282, 416, 515, 557, 572, 665, 713, 729 et 772. PACAUT, 1964, p. 80-81.

113. Annexe, $\mathrm{n}^{\circ}$ III, IV et V.

114. Calcul réalisé à partir du tableau établi par A. Giry (GIRY, 1925, p. 197).

115. Chadd, The ordinal..., p. 1-2.

116. Ibid., p. 2.

117. Ibid., p. 3-4. Gouttebroze, 200o, p. 39-49. Brockhaus, 2009, p. 149. 
Par commodité, nous renvoyons dans cette partie à la numérotation du catalogue des actes de Raoul donné en annexe.

Cette démarche se heurte à quelques difficultés, malgré un assez riche corpus. En effet, la plupart des actes nous sont parvenus via des transcriptions dans les cartulaires de l'abbaye. Ceux-ci ont pour objectif de faciliter la gestion du temporel de l'abbaye; aussi n'y recopie-t-on pas tous les actes de l'abbé, mais plutôt un certain type d'actes. Les contrats de société signés par Raoul d'Argences avec les abbés de Saint-Denis en France et Saint-Lucien de Beauvais, dont nous possédons les originaux, ne figurent ainsi dans aucun des cartulaires ${ }^{118}$. Il faudra donc se méfier d'une surreprésentation impossible à évaluer des actes qui concernent la gestion du temporel. Sur quarante-cinq textes, seuls quatre sont liés à la gestion spirituelle de l'abbaye ${ }^{119} .91 \%$ des actes de notre corpus sont alors liés au temporel: rapport impressionnant, même mis en perspective selon les précautions rappelées ci-dessus.

\section{Les actes liés au spirituel}

On peut diviser ces actes en deux groupes. Deux sont des contrats de societas, dont les originaux sont conservés ${ }^{120}$. Les deux contrats, passés d'une part avec Henri, abbé de Saint-Denis en France et d'autre part avec Evrard, abbé de SaintLucien de Beauvais, présentent un nombre de points communs remarquable. Tous deux datés de novembre 1215, ils ont été établis selon le même modèle diplomatique. La simultanéité peut nous laisser penser que ces contrats de société sont nés d'une volonté de l'abbé de Fécamp. Ceux-ci ont surtout pour vocation d'associer les prières des deux couvents pour les célébrations des défunts. Mais, plus profondément, cette association a dû contribuer à lier Fécamp à deux maisons importantes du domaine royal capétien. Même si le rapprochement du pouvoir capétien n'était peut-être pas le but, le résultat était un plus grand attachement de fait.

Deux actes font part de l'accueil de nouveaux frères: l'un à Fécamp (1211), l'autre dans le prieuré de Saint-Georges de Mantes (1215) ${ }^{121}$. À lire ces deux actes, il semble que la réception d'un frère se faisait sous Raoul conformément au chapitre LVIII de la règle de saint Benoît. Le nouveau frère doit se séparer de tous ses biens, au bénéfice soit des pauvres, soit du monastère où il entre: Pérégrin de Saint-Riquier, qui devient moine de la Trinité de Fécamp, lui abandonne tous ses biens ${ }^{122}$. Le nouveau moine doit ensuite jurer fidélité à

118. Annexe, $\mathrm{n}^{\circ} \mathrm{X}$ et XI.

119. Deux figurent dans les cartulaires (Annexe, $n^{\circ} \mathrm{V}$ et IX); les deux autres non (Annexe, $\mathrm{n}^{\circ} \mathrm{X}$ et XI).

120. Annexe, $\mathrm{n}^{\circ} \mathrm{X}$ et XI. Arch. dép. Seine-Maritime, $7 \mathrm{H} 51$.

121. Annexe, $\mathrm{n}^{\circ} \mathrm{V}$ et IX.

122. Ipse autem pietate dictus se et sua reddidit nobis in morte et in vita. Dedit etiam pro salute anime sue Deo et ecclesie nostre vineam quam habebat in Valle Porte et tres virgatas terre ad vineas faciendas in valle de Merlier inter vineas nostras (Bibl. mun. Rouen, ms 1207 [Y 51], fol. 32). 
l'abbé et l'abbaye - c'est la pétition: ainsi fait Pierre Diacre quand il est reçu dans le prieuré de Saint-Georges de Mantes ${ }^{123}$.

Il est difficile de tirer des conclusions de quatre actes. Cependant, si on les croise avec les travaux de David Chadd concernant la liturgie fécampoise, on peut souligner que Raoul d'Argences a cherché à développer la vie spirituelle de son abbaye, a fortiori si l'on considère qu'il est à l'origine des contrats de sociétété ${ }^{124}$.

\section{Importance des actes liés au temporel}

La bonne gestion du temporel fait partie des missions premières de l'abbé décrites dans la règle de saint Benoit ${ }^{125}$. Quarante-et-un actes sur les quarante-cinq sont liés à l'administration du temporel de l'abbaye. On peut les classer ainsi:

\begin{tabular}{|l|l|l|}
\hline $\begin{array}{l}\text { Concessions } \\
\text { de droits ou de biens }\end{array}$ & 22 actes & $\begin{array}{l}\text { Annexe, } n^{\circ} \text { I, VIII, } \\
\text { XII, XVII, XVIII, XIX, } \\
\text { XXI, XXI, XXIII, XXIV, } \\
\text { XXVII, XXVIII, XXX, } \\
\text { XXXI, XXXII, XXXIV, } \\
\text { XXXV, XXXVI, XXXVII, } \\
\text { XXXVIII, XXXIX, XLI. }\end{array}$ \\
\hline $\begin{array}{l}\text { Confirmations } \\
\text { de biens ou de droits }\end{array}$ & 7 actes & $\begin{array}{l}\text { Annexe, } n^{\circ} \text { VI, XXVI, } \\
\text { XXIX, XXXIII, XLIII, } \\
\text { XLIV, XLV. }\end{array}$ \\
\hline $\begin{array}{l}\text { Confirmations } \\
\text { ou attestations d'actes } \\
\text { d'autres émetteurs }\end{array}$ & 5 actes & $\begin{array}{l}\text { Annexe, } n^{\circ} \text { III, VII, XV, } \\
\text { XVI, XXV. }\end{array}$ \\
\hline Echanges de biens & 3 actes & $\begin{array}{l}\text { Annexe, } n^{\circ} \text { XIII, XIV, } \\
\text { XLII. }\end{array}$ \\
\hline Résolutions de conflit & 2 actes & Annexe, $n^{\circ}$ II, IV. \\
\hline Attributions de fonctions & 2 actes & Annexe, $n^{\circ}$ XX, XL. \\
\hline
\end{tabular}

Des actes pour une continuité

Un nombre assez important d'actes appartient à une logique de continuité dans la gestion de l'abbaye. Plusieurs actes confirment des fiefs ou des vavassories ${ }^{126}$, comme celui-ci daté de 1211: Sciant presentes et futuri quod Radulfus, Dei

123. Ipse vero de fidelitate nobis et successoribus nostris et predicto prioratui exhibenda sollempne prestitit juramentum (Bibl. mun. Rouen, ms 1207 [Y 51], fol. 38-v).

124. Chadd, The ordinal..., p. 1-4.

125. Chapitres II et LXIV.

126. Au sujet des vavassories en Normandie, voir Navel, 1952 et Chanteux, 1990, p. 301-317. 
gratia humilis abbas Fiscannensi, et totus ejusdem loci conventus concessimus et confirmavimus Roberto de Toreville ${ }^{127}$ terram quam avius suus et pater suus tenuerunt de ecclesia Fiscannensi jure hereditario, apud Torevillam, ad tenendum ipsi Roberto et heredibus suis de ecclesia Fiscannensi... ${ }^{128}$. Raoul poursuit les politiques menées par ses prédécesseurs. Dans un cas, il s'agit même d'un vidimus d'une charte d'Henri de Sully, seul exemple dans notre corpus ${ }^{129}$. L'acte d'Henri n'est cependant pas introduit par la formule vidimus, mais ainsi : ... sicut carta inter ecclesiam Fiscannensi et predictos burgenses firmata a tempore Henrici abbatis subsequenter testatur et confirmat: 'Notum sit omnibus tam presentibus quam futuris quod ego Henricus dei gratia abbas... Les témoins de la charte d'Henri de Sully sont omis dans le vidimus ${ }^{130}$ et remplacés par une nouvelle liste: ce vidimus semble donc avoir été établi comme tout autre acte de Raoul, en public, et entouré d'officiers de l'abbaye, dont le cellérier.

\section{Des actes pour une centralisation du pouvoir}

Deux chartes en particulier permettent d'étudier la politique de Raoul, qui est fondée sur une centralisation du pouvoir abbatial. Ces deux actes sont des confirmations de donations faites à l'archidiacre de Fécamp, qui portaient semble-t-il alors le nom de testimonium, terme que l'on trouve au verso d'une des deux chartes en question, dans la formule vraisemblablement écrite par le même scribe que la charte, Richard de Passeor: Testimonium abbatis Fiscanni de masura Willelmi Monachi ${ }^{131}$. Les deux actes, très semblables, suivent le même modèle diplomatique: Universis ad quos presens scriptum pervenerit, Radulphus Dei gratia humilis abbas Fiscannensi, salutem in vero salutari. Noverit universitas vestra quod Radulfus Gernet, burgensis Fiscannensi, dedit et concessit Guillelmo de Argenciis, archidicano Fiscannensi, pro amore et provitio suo, quamdam masuram in vico de Mari... ${ }^{132}$. Ces chartes sont riches de renseignements et suscitent une série de questions. Elles sont d'abord une des traces d'un personnage de l'entourage de l'abbé qui est peu connu: l'archidiacre de Fécamp. Trois sont connus sous Raoul d'Argences: Raoul Peilevilain (dates inconnues) ${ }^{133}$, Guillaume d'Argences (en poste entre 1197 et 1207 au moins) ${ }^{134}$ et Richard d'Argences (en poste entre 1217 et 1230 au moins $)^{135}$. On notera une nouvelle fois la présence de deux hommes d'Argences: signe, s'il en fallait, du népotisme courant sous

\footnotetext{
127. Aujourd'hui Tourville-les-Ifs, dép. Seine-Maritime, cant. Fécamp.

128. Annexe, $n^{\circ}$ VI. Bibl. mun. Rouen, $m s 1207$ [Y 51], fol. 39.

129. Annexe, $n^{\circ}$ XXV. Arch. dép. Seine-Maritime, $7 \mathrm{H} 24$.

130. L'acte d'Henri est conservé: Arch. dép. Seine-Maritime, 7 H 24.

131. Annexe, $n^{\circ}$ XVI. Arch. dép. Seine-Maritime, 7 H 623.

132. Annexe, $n^{\circ}$ XV et XVI. Arch. dép. Seine-Maritime, 7 H 9, p. 5 et 7 H 623.

133. Arch. dép. Seine-Maritime, 7 H 623. Bibl. mun. Rouen, ms 1207 [Y 51], fol. 78v.

134. Arch. dép. Seine-Maritime, 7 H 623; 7 H 9, p. 4 et 5. Bibl. mun. Rouen, ms 1207 [Y 51], fol. 38 bis-v, 39bis, 59v, 6o, 76v, 77-v, 83v, 85 .

135. Arch. dép. Seine-Maritime, 7 H 9, p. 2, 3, 16, 35 et 45. Bibl. mun. Rouen, ms. 1207 [Y 51], fol. 38 bis, $69 \mathrm{v}, 70,74 \mathrm{v}, 81 \mathrm{v}, 88$.
} 
Raoul. Cet officier pose cependant problème par son existence même: Fécamp n'est pas le chef-lieu d'un archidiaconé du diocèse de Rouen. Il semble que ce soit un office créé par l'abbé de Fécamp pour l'assister dans la gestion de l'abbaye: la titulature, en partie usurpatrice, tend à nous faire penser qu'il s'agit d'un clerc, dont les fonctions sont probablement liées à l'exemption de Fécamp ${ }^{136}$. Les abbés de Fécamp ont eu tendance à aspirer à un rang épiscopal, dans les images $^{137}$ et dans les faits ${ }^{138}$. Comme un évêque, il a alors besoin d'officiers qui le secondent, et le comble de l'imitation consiste à leur donner le même nom. Par ces confirmations, Raoul se place comme autorité des deux partis: il confirme les donations en tant que seigneur des donateurs et supérieur du bénéficiaire. C'est un indice de la volonté de contrôle de la ville de Fécamp par le pouvoir abbatial, en particulier après 1204, une fois le pouvoir ducal tombé. Les masures sont placées sous la protection de l'abbaye ${ }^{139}$, et, comme l'a bien montré Annie Renoux, après 1204, les abbés mènent une politique d'achat des anciennes dépendances du château ducal ${ }^{140}$.

La centralisation du pouvoir transparaît également dans des chartes de concession de droits ou de biens. Elles sont les plus nombreuses, représentant la moitié du corpus. Plusieurs de ces chartes nous permettent de mettre en évidence les méthodes de gestion de Raoul. En 1196, il concède à Nicolas d'Estouteville, en échange de son hommage, le droit de patronage de la chapelle de la Mare du Quesnay ${ }^{141}$ située dans la forêt de Fécamp. L'acte est formulé ainsi: Notum sit presentibus et futuris quod ego Radulfus, Dei Gratia humilis abbas Fiscannensi et totus ejusdem loci conventus concessimus et confirmavimus dilecto et familiari nostro Nicholao de Estoteville et heredibus suis, pro servitio et homagio suo: jus patronatus capelle de mara de Caisneio de foresta Fiscannensi. Inde suum recipimus homagium ita quod ipse primo presentabit nobis vicarium suum qui fide prestita sacramento super predicta vicaria nobis primo fidem faciet. Nos vero quoniam alium quam quem prefatus Nicholaus nobis presentaverit ad eandem vicariam presentare nec possumus nec debemus, illum quem nobis presentabit domino Rothomagensi presentabimus ${ }^{142}$. L'acte

136. Au sujet de cette exemption: LEMARIGNIER, 1937, en part. p. 220-225. L'auteur n'évoque cependant pas l'archidiacre de Fécamp.

137. Le sceau de Raoul suit ainsi un modèle épiscopal (AN, J 211, $\mathrm{n}^{\circ} 1$; L 780 (L 1200), $\mathrm{n}^{\circ}$ 58). Voir l'article de M. Bloche en préparation pour ce dossier de Tabularia.

138. L'exemption a été confirmée et précisée à la fin du XII et au XIII ${ }^{\mathrm{e}}$ siècle, donnant naissance à l'« exemption de Fécamp», qui est «l'ensemble des territoires [...] sur lesquels l'exercice de la juridiction spirituelle est retiré à l'archevêque au profit de l'abbé» (LEMARIGNIER, 1937, p. 223).

139. Arch. dép. 7 H 9, p. 5.

140. Renoux, 1991, p. 589-591. Cette politique aboutit pleinement en 1288 par l'achat au roi de Fécamp et au sire d'Estouteville de leurs possessions à Fécamp.

141. Probablement Le Quesnay, hameau de Lamberville (dép. Seine-Maritime, cant. Bacqueville-enCaux).

142. Annexe, $\mathrm{n}^{\circ}$ I. Arch. dép. Seine-Maritime, 7 H 27 : Sachent tous, présents comme futurs, que moi, Raoul, par la grâce de Dieu humble abbé de Fécamp et tout le couvent de ce lieu, avons concédé et confirmé à notre cher familier Nicolas d'Estoteville et à ses héritiers, en échange de son service et son hommage, le droit de patronage de la chapelle de la mare du Quesnay dans la forêt de 
met bien en évidence les jeux de fidélités qui pouvaient guider la gestion de l'abbaye: cette concession d'un droit à un vassal ne va pas sans le maintien d'un contrôle sur le droit de patronage. L'acte précise aussi que les obits du dit Nicolas d'Estouteville et de sa famille seront célébrés à la Trinité de Fécamp. Ce Nicolas n'est autre que l'oncle d'Henri d'Estouteville, chef de la célèbre famille implantée en Normandie et en Angleterre ${ }^{143}$. L'abbé de Fécamp avait donc parmi ses vassaux au moins un membre de la haute noblesse angevine, qui a obtenu un privilège de l'abbé, mais qui reste lié à la Trinité de Fécamp, alors que la maison d'Estouteville est beaucoup plus liée à l'abbaye cistercienne voisine de Notre-Dame de Valmont, au point que ce Nicolas serait devenu frère de Fécamp vers la fin de sa vie ${ }^{144}$.

\section{Conclusions}

Non exhaustive - il faudrait consacrer presque une étude à chaque texte - notre enquête permet toutefois de proposer quelques éléments de conclusion. On ne peut qu'être frappé par la concordance des éléments qui donnent l'impression d'une politique de codification sous Raoul d'Argences. L'étude diplomatique montre que les actes se ressemblent de plus en plus, surtout après 1204 . Le contenu des actes montre aussi une volonté de contrôle de plus en plus grande de la part de l'abbé de Fécamp.

Devenu royal en 1204 comme tous les abbés bénédictins ${ }^{145}$, il figure parmi ceux qui sont les plus liés à la monarchie capétienne au XIII ${ }^{\mathrm{e}}$ siècle (contrat de société avec Saint-Denis, obtention de privilèges, etc.). Il acquiert même une réputation dans l'ensemble du royaume sous Louis IX: Guillaume Vaspail est appelé en 1241 au concile devant se tenir à Rome contre l'Empereur Frédéric II et fait donc partie des prélats enlevés à Pise par le roi de Sardaigne (le fils de Frédéric) ${ }^{146}$; dans les textes, il fait jeu égal avec les abbés de Cluny, Cîteaux, Clairvaux et la Piété-Dieu ${ }^{147}$.

Fécamp. Nous avons donc reçu son hommage aux conditions suivantes: lui-même nous présentera d'abord le vicaire de son choix, qui après avoir donné sa foi par serment pour cette vicairie susdite, nous jurera fidélité en premier. Quant à nous, puisque nous ne pouvons ni ne devons présenter à cette même vicairie un autre que celui que le susdit Nicolas nous présentera, nous présenterons à l'archevêque de Rouen celui qu'il nous aura présenté. (Trad. F. Paquet)

143. De la Morandière, 1903, p. 70-71.

144. De la Morandière, 1903, en part. p. 38-44, 56-62 et 70-77.

145. Baldwin (éd.), Les registres de Philippe Auguste, p. 326-327.

146. Delisle (éd.), "E chronico abbatum fiscannensium», p. 431. Il aurait été libéré avec l'aide d'un archidiacre de Bayeux, sans payer aucune rançon (ibid., p. 431). Au sujet de ce concile: Hefele (éd.), Histoire des conciles..., t. VIII, p. 323-326; pour la place des français: Berger, 1893, p. 5-6.

147. Cette liste figure dans une lettre de Grégoire IX: Rinaldi (éd.), Annales Ecclesiastici, 1870, t. XXI (1229-1256), nº 69, p. 249; HuIllard-Breholles (éd.), Historia diplomatica Friderici secundi, t. V, vol. 2, p. 1136-1138. On dispose aussi d'une lettre des abbés de Cîteaux, Clairvaux et la PiétéDieu, faisant part de leur capture à l'abbé de Savigny, dans laquelle des nouvelles de l'abbé de Fécamp sont données: ibid., t. V, vol. 2, p. 1121-1122. 
À une autre échelle, l'abbé est un seigneur - le terme «dominus» devient très courant dans les chartes et notices à partir de Raoul d'Argences ${ }^{148}$ - à la tête d'un domaine d'une grande importance. L'ensemble des terres désignées sous le terme d'" exemption de Fécamp » est indépendante des pouvoirs ecclésiastiques (exemption) et temporels locaux (haute justice et abbaye royale). Pour le roi, il y a le risque de créer une institution indépendante très forte, qui tient un des principaux ports de la Normandie, mais l'abbé de Fécamp ayant fait la preuve de sa fidélité au Capétien, lui confier le port et la ville totalement revient à s'assurer que ce port ne retombe pas dans les mains angevines... Cette concession contribue aussi à éliminer les institutions communales de Fécamp que Jean sans Terre a mises en place en $1202^{149}$. La commune est subordonnée à l'abbaye, et les bourgeois ne parviennent pas à se faire attribuer les coutumes du port, à savoir le monopole de la vente de poissons ${ }^{150}$. Après avoir vécu sous la tutelle de Rouen, la commune de Fécamp disparait après $1235^{151}$. Philippe Auguste a préféré l'abbé à la commune. À travers ce dernier exemple, on voit clairement que l'histoire d'un abbatiat doit aussi être politique, et que le croisement des différentes échelles de pouvoir permet à la fois de mieux saisir le fonctionnement du pouvoir abbatial, ainsi que la période de rattachement de la Normandie à la couronne capétienne: la naissance d'une province française.

\section{Sources}

\section{Sources manuscrites}

Arch. dép. Seine-Maritime, $7 \mathrm{H} 9$ (cartulaire de la Trinité de Fécamp). Arch. dép. Seine-Maritime, $7 \mathrm{H}$ (abbaye de la Trinité de Fécamp).

Bibl. mun. Rouen, ms 1207 [Y 51] (cartulaire de la Trinité de Fécamp).

\section{Sources éditées}

Baldwin, John (éd.), Les registres de Philippe Auguste, Paris, Diffusion de Boccard, Recueil des Historiens de France: documents financiers et administratifs, t. VII, 1992.

Chadd, David (éd.), The ordinal of the Abbey of Holy Trinity Fécamp (Musée de la Bénédictine, ms 168), Woodbridge, Boydell Press, 1999-2002, 2 vol.

Delisle, Léopold (éd.), Cartulaire normand de Philippe-Auguste, Louis VIII, Saint Louis et Philippe le Hardi, (Mémoires de la Société des Antiquaires de Normandie, t. XVI), Caen, Hardel, 1852.

148. Ceci n'est cependant pas propre à Fécamp; on le rencontre par exemple aussi à Jumièges (Arch. dép. Seine-Maritime, $9 \mathrm{H} 4$ et $9 \mathrm{H}$ 5).

149. Rotuli Litterarum Patentium, p. 13-14.

150. Bibl. mun. Rouen, ms 1207 [Y 51], fol. 94.

151. DECK, 1960, p. 323-324. 
Delisle, Léopold (éd.) et alii, «E chronico abbatum fiscannensium », in Recueil des historiens de la Gaule et de la France, t. XXIII, Paris, 1894, p. 430-432.

Delisle, Léopold (éd.), Recueil des actes d'Henri II, roi d'Angleterre et duc de Normandie concernant les provinces françaises et les affaires de France. Introduction, Paris, Imprimerie Nationale, 1909.

Hardy, Thomas Duffus (éd.), Rotuli litterarum patentium in Turri Londinensi, Londres, Eyre and Spottiswoode, 1835.

Hefele, Carl Joseph (éd.), Histoire des conciles d'après les documents originaux, Paris, Letouzey et Ané, 1907-1952, 21 vol.

Huillard-Breholles, Alphonse (éd.), Historia diplomatica Friderici secundi sive constitutionis, privilegia, mandata, instrumenta quae supersunt istius imperatoris et filiorum ejus, Paris, Plon, 1852-1861, 12 vol.

Luchaire, Achille (éd.), Étude sur les actes de Louis VII, Paris, Picard, 1885.

Ramackers, Johannes (éd.), Papsturkunden in Frankreich, 2. Normandie, Vandenhoeck und Rupprecht, Göttingen, 1937.

Rinaldi, Oderico (éd.), Annales Ecclesiastici, Rome, 1846-1863, rééd. Paris, V. Palme, 1864-1883, 37 vol.

Stapleton, Thomas (éd.), Magni rotuli scaccarii Normanniae ab regibus Angliae, Londres, Société des Antiquaires de Londres, 1840.

Teulet, Alexandre (éd.), Layettes du Trésor des Chartes, Paris, Plon, 1863, 5 vol.

\section{Bibliographie}

Atsma, Harmut et Vezin, Jean, «Les responsables de la transcription des actes juridiques et les services de l'écriture au $\mathrm{X}^{\mathrm{e}}$ siècle: l'exemple de Cluny", in Le statut du scripteur au Moyen Âge (Actes du XIIe colloque scientifique du Comité international de paléographie latine), Marie-Clothilde Hubert, Emmanuel Poulle et Marc H. Sмiтh, (éd.), Paris, École nationale des Chartes, 200o, p. 9-15.

Baldwin, John, Philippe Auguste et son gouvernement: les fondations du pouvoir politique en France au Moyen Âge, Berkeley, University of California Press, 1986; Paris, Fayard, 1991 pour la traduction française.

Beaurepaire (de), François, Les noms de communes et anciennes paroisses de SeineMaritime, Paris, Picard, 1979.

Berger, Elie, Saint-Louis et Innocent IV. Etude sur les rapports de la France et $d u$ Saint-Siège, Paris, Thorin et fils, 1893.

Bloche, Michaël, «Les suscriptions dans les actes des abbés de Fécamp (XIe-début du $\mathrm{XIV}^{\mathrm{e}}$ siècle)», Tabularia «Études ", $\mathrm{n}^{\circ} 11$, en préparation.

Brocknaus, Katrin, L'abbatiale de la Trinité de Fécamp et l'architecture normande au Moyen Âge, Caen, Société des antiquaires de Normandie, 2009.

Chanteux, Henry, «Les vavassories normandes et le problème de leurs origines ", in Recueil d'études en hommage à Lucien Musset, Cahier des Annales de Normandie, $\mathrm{n}^{\circ}$ 23, Caen, 1990, p. 301-317.

Deck, Suzanne, «Formation des communes en Haute-Normandie et communes éphémères ", Annales de Normandie, 1960, $\mathrm{n}^{\circ} 3$, p. 207-227 et $\mathrm{n}^{\circ}$ 4, p. 317-329. 
De La Morandière, Gabriel, Histoire de la Maison d'Estouteville, Paris, Ch. Delagrave, 1903.

Delisle, Léopold, «Philippe Auguste et Raoul d’Argences, abbé de Fécamp», Bibliothèque de l'Ecole des Chartes, t. LXV, 1904, p. 390-397.

Fallue, Léon, Histoire de la ville et de l'abbaye de Fécamp, Rouen, Périeux, 1841.

Gazeau, Véronique, Normannia Monastica, t. I, Princes normands et abbés bénédictins et $t$. II, Prosopographie des abbés bénédictins ( $X^{e}-X I I^{e}$ siècle), Caen, Publications du CRAHM, 2007.

Geshin de Bourgogne, Jules et Barthélémy (de), Anatole, Anciens évêchés de Bretagne. Histoire et monument, t. 3, diocèse de Saint-Brieuc, Paris, Hérold et Saint-Brieuc, Guyon Frères, 1864.

GIRY, Alfred, Manuel de diplomatique, Paris, Felix Alcan, 1925.

Gourdon de Genouillac, Henri, Histoire de l'abbaye de Fécamp et de ses abbés, Fécamp, Marinier, 1875.

Gouttebroze, Jean-Guy, Le précieux sang de Fécamp. Origine et développement d'un mythe chrétien, Paris, Honoré Champion, 2000.

Guyotjeannin, Olivier, Pyскe, Jacques et Tock, Benoît-Michel, Diplomatique médiévale, Turnhout, Brepols, «L’atelier du médiéviste» $\mathrm{n}^{\circ} 2,1993$.

Guyoyjeannin, Olivier et Tock, Benoît-Michel, «Mos presentis patrie: les styles de changement du millésime dans les actes français (XI ${ }^{\mathrm{e}}-\mathrm{XVI}^{\mathrm{e}}$ siècles)», Bibliothèque de l'École des Chartes, t. 157, 1999, livraison 1, p. 41-109.

Lemarignier, Jean-François, Étude sur les privilèges d'exemption et de juridiction des abbayes normandes, Paris, Picard, 1937.

Leroux DE Lincy, Antoine, Essai historique et littéraire sur l'abbaye de Fécamp, Rouen, Périaux, 1840.

Mabille, Micheline, Le temporel de l'abbaye de Fécamp des origines à la fin du $X I I I^{e}$ siècle, thèse de l'Ecole nationale des Chartes, soutenue le 26 mars 1953 à Paris (Arch. nat., AB XXVIII, 126).

Musset, Lucien, "Quelques problèmes posés par l'annexion de la Normandie au domaine royal français", in La France de Philippe-Auguste: le temps des mutations, Robert-Henri Bautier (dir.), (Actes du colloque international du CNRS de 1980), Paris, Éditions du CNRS, 1982.

Navel, Henri, «Recherches sur les institutions féodales en Normandie (Région de Caen)», Bulletin de la Société des antiquaires de Normandie, tome LI, Caen et Rouen, 1952, p. 5-176.

PACAUt, Marcel, Louis VII et son royaume, Paris, SEVPEN, 1964.

Petit-Dutaillis, Charles, «Querimoniae Normannorum», in Essays in Medieval History presented to Thomas Frederick Tout, Little, Andrew G., et Powicke, Maurice (éd.), Manchester, 1925.

Power, Daniel J., The Norman Frontier in the twelfth and early thirteenth centuries, Cambridge, Cambridge University Press, 2004.

Powicke, Maurice, The loss of Normandy (1189-1204). Studies in the Story of the Angevin Empire, Manchester, Manchester University Press, 1913, rééd. 1961. 
Renoux, Annie, Fécamp, du palais ducal au palais de Dieu. Bilan historique et archéologique des recherches menées sur le site du château des ducs de Normandie (II ${ }^{e}$ s. av. J.-C.-XVIII ${ }^{e}$ s.), Paris, éditions du CNRS, 1991.

Rosny (de), Joseph, Tableau littéraire de la France pendant le XIII siècle ou recherches historiques sur la situation des arts, sciences et belles-lettres depuis l'an 1200 jusqu'en 1301, Paris, Désiré Hécart, 1809.

Roukt, Dominique (éd.), Le cartulaire de l'abbaye bénédictine de Saint-Pierre de Préaux (1034-1227), Paris, éditions du Comité des travaux historiques et scientifiques, Collection des documents inédits sur l'histoire de France, section d'histoire et de philosophie des civilisations médiévales, vol. XXXIV, 2005.

Schmitz, Philibert, Histoire de l'ordre de Saint Benoît, Les éditions de Maredsous, 7 vol., 1942-1956.

Six, Manon, «Le prieuré de Saint-Gabriel », Annales de Normandie, 2002, n 2, p. 99-127 et $\mathrm{n}^{\circ} 3$, p. 225-251.

Tock, Benoît-Michel, Scribes, souscripteurs et témoins dans les actes privés en France (VII ${ }^{e}$-début du XII siècle), Turnhout, Brepols, 2005.

\section{Annexe: catalogue des actes de Raoul d'Argences ${ }^{152}$}

\section{I}

1196 - Raoul d'Argences concède à Nicolas d'Estouteville, en échange de son hommage, le droit de patronage de la chapelle de la Mare du Quesnay ${ }^{153}$ située dans la forêt de Fécamp.

A : Original: Arch. dép. Seine-Maritime, 7 H 27.

\section{II}

1201 - Raoul d'Argences fait part de la résolution du conflit qui s'était élevé entre la Trinité de Fécamp et Notre-Dame de Mantes ${ }^{154}$ au sujet du clos Pierre.

$A$ : Original perdu.

$B$ : Copie d'après $A, \mathrm{XIII}^{\mathrm{e}}-\mathrm{XIV}^{\mathrm{e}}$ siècle: cartulaire, Bibl. mun. Rouen, ms 1207

[Y 51], fol. 31v.

$C$ : Copie d'après $B$, fin $\mathrm{XVI}^{\mathrm{e}}$ siècle: BnF, nouv. acq. lat., ms 2412, $\mathrm{n}^{\circ}$ 91, p. 72.

152. Ce catalogue ne peut être qu'incomplet. Nous y avons référencé tous les actes que nous avons rencontrés jusqu'à présent. Toutes les dates données sont en nouveau style (cf. 1.3.).

153. Probablement Le Quesnay, hameau de Lamberville (dép. Seine-Maritime, cant. Bacqueville-enCaux).

154. Notre-Dame de Mantes: abbaye de chanoines réguliers, sise à Mantes-la-Jolie (dép. Yvelines, chef-lieu de cant.). L'abbaye de Fécamp possède un prieuré à Mantes, Saint-Georges. 
Janvier 1205 - Raoul d'Argences atteste que ses hommes de Boissy ${ }^{155}$ doivent s'acquitter d'une redevance annuelle comme droit de tensement, pour l'obtention de la protection royale pour ce village.

A: Original: Trésor des Chartes, J 211, Normandie, II, 1.

$B$ : Copie d'après $A$ : registre normand de la Chambre des Comptes, BnF, ms lat. 9067 , fol. $96 \mathrm{v}$.

$a$ : Teulet, Layettes..., t. I, $\mathrm{n}^{\circ}$ 744, p. 281. - $\boldsymbol{b}$ : Delisle, 1904, p. 392.

IV

Janvier 1209 - Raoul d'Argences et Henri d'Estouteville font part de la résolution de la querelle qui les opposait au sujet du moulin Bidoille.

$A$ : Original perdu.

$B$ : Copie d'après $A, \mathrm{XIII}^{\mathrm{e}}-\mathrm{XIV}^{\mathrm{e}}$ siècle: cartulaire, Bibl. mun. Rouen, ms 1207 [Y 51], fol. 63-v.

$C$ : Copie d'après $B$, fin $\mathrm{XVI}^{\mathrm{e}}$ siècle: BnF, nouv. acq. lat., $\mathrm{ms} 2412, \mathrm{n}^{\circ} 209$, p. 190192.

V

19 février 1211 - Raoul d'Argences fait part de l'accueil de Pérégrin de Saint-Riquier comme moine de la Trinité de Fécamp. Celui-ci abandonne ses biens à l'abbaye.

$A$ : Original perdu.

$B$ : Copie d'après $A, \mathrm{XIII}^{\mathrm{e}}-\mathrm{XIV}^{\mathrm{e}}$ siècle: cartulaire, Bibl. mun. Rouen, ms 1207 [Y 51], fol. 32.

$C$ : Copie d'après $B$, fin $\mathrm{XVI}^{\mathrm{e}}$ siècle: BnF, nouv. acq. lat., ms 2412, $\mathrm{n}^{\circ}$ 93, p. 73-74.

VI

1211 - Raoul d'Argences confirme à Robert de Tourville ${ }^{156}$ une terre située à Tourville que son père et son aïeul tenaient déjà de l'abbé de Fécamp.

$A$ : Original perdu.

$B$ : Copie d'après $A, \mathrm{XIII}^{\mathrm{e}}-\mathrm{XIV}^{\mathrm{e}}$ siècle: cartulaire, Bibl. mun. Rouen, ms 1207 [Y 51], fol. 39.

$C$ : Copie d'après $B$, fin $X_{V I}{ }^{e}$ siècle: BnF, nouv. acq. lat., ms 2412, $\mathrm{n}^{\circ} 120$, p. 92-93.

VII

1211 - Raoul d'Argences atteste que Philippe Auguste, roi de France, lui a concédé le droit de haute justice.

155. Dép. Yvelines, cant. Bonnières-sur-Seine, c. Boissy-Mauvoisin. 156. Aujourd'hui Tourville-les-Ifs, dép. Seine-Maritime, cant. Fécamp.

http://www.unicaen.fr/mrsh/craham/revue/tabularia/print.php?dossier=dossier11\&file=02paquet.xml 
$A$ : Original: Trésor des chartes, J 211, Normandie, II, 3.

$B$ : Copie d'après $A$ : registre normand de la Chambre des Comptes, BnF, ms lat. 9067 , fol. $97 \mathrm{v}$.

$a$ : Delisle, Cartulaire normand..., $\mathrm{n}^{\circ}$ 216, p. 34 et 299.

\section{VIII}

1213 - Raoul d'Argences et le couvent de Fécamp concèdent en fief à Richard de Petra, de Barentin ${ }^{157}$, une masure et une terre qui a été donnée au prieuré Saint-Gervais ${ }^{158}$.

$A$ : Original perdu.

$B$ : Copie d'après $A, \mathrm{XIII}^{\mathrm{e}}$-XIV $^{\mathrm{e}}$ siècle: cartulaire, Bibl. mun. Rouen, ms 1207 [Y 51$],$ fol. 31 .

$C$ : Copie d'après $B$, fin XVI ${ }^{e}$ siècle: BnF, nouv. acq. lat., ms 2412, $\mathrm{n}^{\circ}$ 90, p. 71-72.

IX

1215 - Raoul d'Argences informe de la réception comme frère de Pierre Diacre, dans le prieuré Saint-Georges de Mantes.

$A$ : Original perdu.

$B$ : Copie d'après $A, X I I I^{e}-X_{V} V^{e}$ siècle: cartulaire, Bibl. mun. Rouen, ms 1207 [Y 51], fol. 38-v.

$C$ : Copie d'après $B$, fin $\mathrm{XVI}^{\mathrm{e}}$ siècle: $\mathrm{BnF}$, nouv. acq. lat., ms 2412, $\mathrm{n}^{\circ} 117$, p. $90-91$.

\section{$\mathrm{X}$}

Novembre 1215 - Contrat de société entre la Trinité de Fécamp et Saint-Denis en France, émis par Raoul d'Argences, abbé de Fécamp et Henri, abbé de Saint-Denis.

$A$ : Original: Arch. dép. Seine-Maritime, 7 H 51.

XI

Novembre 1215 - Contrat de société entre la Trinité de Fécamp et Saint-Lucien de Beauvais ${ }^{159}$, émis par Raoul d'Argences, abbé de Fécamp et Évrard, abbé de Saint-Lucien.

$A$ : Original: Arch. dép. Seine-Maritime, 7 H 51.

XII

1216 - Raoul d'Argences concède à maître Guillaume de Saint-Meulan un bien relevant de l'église de Saint-Gervais ${ }^{160}$.

157. Dép. Seine-Maritime, cant. Pavilly.

158. Prieuré dépendant de la Trinité de Fécamp et situé à Rouen.

159. Dép. Oise, chef-lieu de cant.

160. Prieuré de la Trinité de Fécamp situé à Rouen. 
$A$ : Original perdu.

$B$ : Copie d'après $A, \mathrm{XIII}^{\mathrm{e}}$-XIV $^{\mathrm{e}}$ siècle: cartulaire, Bibl. mun. Rouen, ms 1207

[Y 51], fol. 32v, 33 .

$C$ : Copie d'après $B$, fin $\mathrm{XVI}^{e}$ siècle: BnF, nouv. acq. lat., $\mathrm{ms} 2412, \mathrm{n}^{\circ}$ 95, p. 75.

\section{XIII}

Novembre 1217 - Raoul d'Argences et Nicolas, prieur de la Madeleine de Rouen font part d'un échange de rentes entre leurs deux couvents.

A: Original: Arch. dép. Seine-Maritime, 7 H 2095.

\section{XIV}

1219 - Raoul d'Argences, abbé, échange avec Raoul d'Argences, chevalier, les droits que celui-ci possédait dans la forêt d'Argences, contre le bois Trossele entre Héritot ${ }^{161}$ et le Long Mesnil ${ }^{162}$.

A : Original: Arch. dép. Calvados, H 4368.

\section{XV}

1190-1217 - Raoul d'Argences confirme la donation d'une masure à Guillaume d'Argences, archidiacre de Fécamp, par Raoul Gernet, bourgeois de Fécamp.

$A$ : Original perdu.

$B$ : Copie d'après $A, X V^{e}$ siècle : cartulaire, Arch. dép. Seine-Maritime 7 H 9, p. 5.

\section{XVI}

1190-1217 - Raoul d'Argences confirme la donation d'une masure à Guillaume d'Argences, archidiacre de Fécamp, par Guillaume le Moine, fils de Roger. Raoul est le garant de cette donation.

$A$ : Original: Arch. dép. Seine-Maritime, 7 H 623.

$B$ : Copie d'après $A$, XIV ${ }^{e}$ siècle: cartulaire, Arch. dép. Seine-Maritime 7 H 9, p. 5-6.

\section{XVII}

1204-1219 - Raoul d'Argences concède, à la demande de Philippe Auguste, une vavassorie située à Heudebouville ${ }^{163}$ à Richard Syme, serviteur du roi de France.

$A$ : Original perdu.

$B$ : Copie d'après $A, \mathrm{XIII}^{\mathrm{e}}$-XIV $^{\mathrm{e}}$ siècle: cartulaire, Bibl. mun. Rouen, ms 1207 [Y 51], fol. 34 .

161. Hameau dépendant de Saint-Ouen-du-Mesnil-Oger (dép. Calvados, cant. Troarn).

162. Peut-être le hameau «Le Mesnil» de la même commune actuelle.

163. Dép. Eure, cant. Louviers.

http://www.unicaen.fr/mrsh/craham/revue/tabularia/print.php?dossier=dossier11\&file=02paquet.xml 
$C$ : Copie d'après $B$, fin XVI ${ }^{e}$ siècle: BnF, nouv. acq. lat., ms 2412, ${ }^{\circ}$ 99, p. 78.

\section{XVIII}

1190-1219 - Raoul d'Argences et le couvent de Fécamp concèdent en fief à Bernard, fils du prévôt de Tourville ${ }^{164}$, quatre acres et une vergée de terre dans le Bois Laurent, que le même Bernard a défrichées à la demande de l'abbé.

$A$ : Original perdu.

$B$ : Copie d'après $A, \mathrm{XIII}^{\mathrm{e}}-\mathrm{XIV}^{\mathrm{e}}$ siècle: cartulaire, Bibl. mun. Rouen, ms 1207 [Y 51], fol. 31 .

$C$ : Copie d'après $B$, fin $\mathrm{XVI}^{e}$ siècle: $\mathrm{BnF}$, nouv. acq. lat., ms 2412, $\mathrm{n}^{\circ}$ 89, p. $70-71$.

\section{XIX}

1190-1219 - Raoul d'Argences concède à Richard de Mézières des terres situées à Mézières ${ }^{165}$.

$A$ : Original perdu.

$B$ : Copie d'après $A, \mathrm{XIII}^{\mathrm{e}}-\mathrm{XIV}^{\mathrm{e}}$ siècle: cartulaire, Bibl. mun. Rouen, ms 1207 [Y 51], fol. 31v, 32.

$C$ : Copie d'après $B$, fin $X_{V I}{ }^{e}$ siècle: BnF, nouv. acq. lat., ms 2412, $n^{\circ}$ 92, p. 73.

\section{XX}

119o-1219 - Raoul, abbé de Fécamp, nomme Robert Chambrier à l'office de boulanger, pour son service, en remplacement de Guillaume.

$A$ : Original perdu.

$B$ : Copie d'après $A, \mathrm{XIII}^{\mathrm{e}}$-XIV $^{\mathrm{e}}$ siècle: cartulaire, Bibl. mun. Rouen, ms 1207 [Y 51], fol. 32-v ${ }^{166}$.

\section{XXI}

1190-1219 - Raoul d'Argences concède à Guillaume de Dune et à sa femme Christiane une terre à Boleranum ${ }^{167}$ qui a appartenu à Bentia de la Haretelen.

$A$ : Original perdu.

$B$ : Copie d'après $A, \mathrm{XIII}^{\mathrm{e}}-\mathrm{XIV}^{\mathrm{e}}$ siècle : cartulaire, Bibl. mun. Rouen, ms 1207 [Y 51], fol. 32-v.

$C$ : Copie d'après $B$, fin XVI ${ }^{e}$ siècle: BnF, nouv. acq. lat., ms 2412, $n^{\circ}$ 94, p. 74-75.

\footnotetext{
164. Aujourd'hui Tourville-les-Ifs, dép. Seine-Maritime, cant. Fécamp.

165. Dép. Yvelines, cant. Guerville, c. Mézières-sur-Seine.

166. Cet acte n'est pas recopié dans la copie de la fin du XVI siècle du cartulaire (BnF, nouv. acq. lat., ms 2412).

167. Non identifié
} 


\section{XXII}

1190-1219 - Raoul d'Argences cède à Richard, fils de Guillaume Roscelin, et à sa femme un grenier situé à Argences ${ }^{168}$.

$A$ : Original perdu.

$B$ : Copie d'après $A, \mathrm{XIII}^{\mathrm{e}}$-XIV $^{\mathrm{e}}$ siècle: cartulaire, Bibl. mun. Rouen, ms 1207

[Y 51$]$, fol. 33 .

$C$ : Copie d'après $B$, fin $\mathrm{XVI}^{\mathrm{e}}$ siècle: BnF, nouv. acq. lat., $\mathrm{ms} 2412, \mathrm{n}^{\circ}$ 96, p. 76.

\section{XXIII}

119o-1219 - Raoul d'Argences concède des biens, notamment des vignes, à Léon Jemello, bourgeois de Mantes ${ }^{169}$.

$A$ : Original perdu.

$B$ : Copie d'après $A, \mathrm{XIII}^{\mathrm{e}}{ }^{-\mathrm{XIV}}{ }^{\mathrm{e}}$ siècle: cartulaire, Bibl. mun. Rouen, ms 1207 [Y 51], fol. 33-v.

$C$ : Copie d'après $B$, fin $\mathrm{XVI}^{\mathrm{e}}$ siècle: $\mathrm{BnF}$, nouv. acq. lat., ms 2412, $\mathrm{n}^{\circ}$ 97, p. 76-77.

\section{XXIV}

1190-1219 - Raoul d'Argences concède à Richard de Mézières ${ }^{170}$ des terres situées à La Sablonnière ${ }^{171}$.

$A$ : Original perdu.

$B$ : Copie d'après $A, \mathrm{XIII}^{\mathrm{e}}$-XIV$^{\mathrm{e}}$ siècle: cartulaire, Bibl. mun. Rouen, ms 1207 [Y 51], fol. 33v.

$C$ : Copie d'après $B$, fin XVI ${ }^{e}$ siècle: BnF, nouv. acq. lat., ms $2412, n^{\circ}$ 98, p. 77-78.

\section{XXV}

1190-1219 - Raoul d'Argences vidime une charte d'Henri de Sully ${ }^{172}$, son prédécesseur, concernant Rye ${ }^{173}$, dans laquelle il fixe les droits de coutume dus à l'abbé par les navires de pêche pratiquant ce port.

$A$ : Original: Arch. dép. Seine-Maritime, $7 \mathrm{H} 24$.

$B$ : Copie d'après $A, \mathrm{XIII}^{\mathrm{e}}{ }^{-\mathrm{XIV}}{ }^{\mathrm{e}}$ siècle: cartulaire, Bibl. mun. Rouen, ms 1207 [Y 51], fol. 34v.

$C$ : Copie d'après $B$, fin $\mathrm{XVI}^{\mathrm{e}}$ siècle: $\mathrm{BnF}$, nouv. acq. lat., $\mathrm{ms} 2412, \mathrm{n}^{\circ} 100$, p. 78-79.

\footnotetext{
168. Dép. Calvados, cant. Troarn.

169. Dép. Yvelines, c. Mantes-la-Jolie, chef-lieu de cant.

170. Dép. Yvelines, cant. Guerville, c. Mézières-sur-Seine.

171. Dép. Eure, cant. Vernon, c. Douains.

172. Henri de Sully est abbé de Fécamp de 1139 à 1187.

173. Sussex.
}

http://www.unicaen.fr/mrsh/craham/revue/tabularia/print.php?dossier=dossier11\&file=02paquet.xml 


\section{XXVI}

1190-1219 - Raoul d'Argences confirme une masure située à Aizier ${ }^{174}$ à Gauthier, fils d'Odon de Perron.

$A$ : Original perdu.

$B$ : Copie d'après $A, \mathrm{XIII}^{\mathrm{e}}-\mathrm{XIV}^{\mathrm{e}}$ siècle: cartulaire, Bibl. mun. Rouen, ms 1207 [Y 51], fol. 35 .

$C$ : Copie d'après $B$, fin $X_{V I}{ }^{e}$ siècle: $B n F$, nouv. acq. lat., ms $2412, \mathrm{n}^{\circ} 102, \mathrm{p} .81$.

\section{XXVII}

1190-1219 - Raoul d'Argences concède un tènement à un de ses serviteurs, Hervé d'Amblie ${ }^{175}$.

$A$ : Original perdu.

$B$ : Copie d'après $A, \mathrm{XIII}^{\mathrm{e}}-\mathrm{XIV}^{\mathrm{e}}$ siècle: cartulaire, Bibl. mun. Rouen, ms 1207 [Y 51], fol. 35 .

$C$ : Copie d'après $B$, fin $\mathrm{XVI}^{\mathrm{e}}$ siècle: BnF, nouv. acq. lat., ms 2412, $\mathrm{n}^{\circ}$ 103, p. 81-82.

\section{XXVIII}

1190-1219 - Raoul d'Argences concède une masure à Odon Loce et ses frères.

$A$ : Original perdu.

$B$ : Copie d'après $A, \mathrm{XIII}^{\mathrm{e}}$-XIV ${ }^{\mathrm{e}}$ siècle: cartulaire, Bibl. mun. Rouen, ms 1207 [Y 51], fol. 35-v.

$C$ : Copie d'après $B$, fin XVI e siècle: BnF, nouv. acq. lat., ms 2412, ${ }^{\circ} 104$, p. 82.

\section{XXIX}

1190-1219 - Raoul d'Argences confirme à Thomas, fils de Ranoulf de Ryes, des terres situées à Ryes ${ }^{176}$ et Mondeville ${ }^{177}$.

$A$ : Original perdu.

$B$ : Copie d'après $A, \mathrm{XIII}^{\mathrm{e}}$-XIV $^{\mathrm{e}}$ siècle: cartulaire, Bibl. mun. Rouen, ms 1207 [Y 51], fol. 35v.

$C$ : Copie d'après $B$, fin $\mathrm{XVI}^{\mathrm{e}}$ siècle: BnF, nouv. acq. lat., ms 2412, $\mathrm{n}^{\circ}$ 105, p. 82-83.

\section{XXX}

1190-1219 - Raoul d'Argences concède un tènement à Robert de Villerville ${ }^{178}$.

\footnotetext{
174. Dép. Eure, cant. Quillebeuf-sur-Seine.

175. Dép. Calvados, cant. Creully.

176. Dép. Calvados, chef-lieu de cant.

177. Dép. Calvados, cant. Caen.

178. Dép. Calvados, Trouville-sur-Mer.
} 


\begin{abstract}
$A$ : Original perdu.
$B$ : Copie d'après $A, \mathrm{XIII}^{\mathrm{e}}-\mathrm{XIV}^{\mathrm{e}}$ siècle: cartulaire, Bibl. mun. Rouen, ms 1207

[Y 51], fol. $35 \mathrm{v}$.

$C$ : Copie d'après $B$, fin XVI e siècle: BnF, nouv. acq. lat., ms 2412, $n^{\circ} 106$, p. 83.
\end{abstract}

\title{
XXXI
}

1190-1219 - Raoul d'Argences concède à Guillaume, fils d'Herbert, et à Renaud, son fils, un moulin à Staninges ${ }^{179}$, à charge pour eux de fournir le blé nécessaire aux moines dépendant de Fécamp dans ce baillage.

$A$ : Original perdu.

$B$ : Copie d'après $A, \mathrm{XIII}^{\mathrm{e}}-\mathrm{XIV}^{\mathrm{e}}$ siècles: cartulaire, Bibl. mun. Rouen, ms 1207 [Y 51], fol. 35v, 36 .

$C$ : Copie d'après $B$, fin XVI ${ }^{e}$ siècle: BnF, nouv. acq. lat., ms 2412, $\mathrm{n}^{\circ}$ 107, p. 83-84.

\section{XXXII}

119o-1219 - Raoul d'Argences concède à Renaud de Brueria ${ }^{180}$ et à sa femme, Basilia, une masure située près de l'hospice de l'abbaye de Fécamp.

$A$ : Original perdu.

$B$ : Copie d'après $A, \mathrm{XIII}^{\mathrm{e}}-\mathrm{XIV}^{\mathrm{e}}$ siècles: cartulaire, Bibl. mun. Rouen, ms 1207 [Y 51], fol. 36 .

$C$ : Copie d'après $B$, fin XVI ${ }^{e}$ siècle: BnF, nouv. acq. lat., ms $2412, \mathrm{n}^{\circ} 108$, p. 84.

\section{XXXIII}

1190-1219 - Raoul d'Argences confirme à Geoffroi de Rapendonia ${ }^{181}$ un pré situé à Mondeville ${ }^{182}$.

$A$ : Original perdu.

$B$ : Copie d'après $A, \mathrm{XIII}^{\mathrm{e}}-\mathrm{XIV}^{\mathrm{e}}$ siècles: cartulaire, Bibl. mun. Rouen, ms 1207 [Y 51], fol. 36 .

$C$ : Copie d'après $B$, fin XVI ${ }^{e}$ siècle: BnF, nouv. acq. lat., ms 2412, $\mathrm{n}^{\circ}$ 109, p. 84-85.

\section{XXXIV}

1190-1219 - Raoul d'Argences concède à Richard de Mézières ${ }^{183}$ une terre située à La Sablonnière ${ }^{184}$.

\footnotetext{
179. Aujourd'hui Steyning (Sussex). Église dépendant de Fécamp depuis une donation de Guillaume le Conquérant en 1085 (Arch. dép. Seine-Maritime, 7 H 2151).

180. Non identifié

181. Non identifié.

182. Dép. Calvados, cant. Caen.

183. Dép. Yvelines, cant. Guerville, c. Mézières-sur-Seine.

184. Dép. Eure, cant. Vernon, c. Douains.
}

http://www.unicaen.fr/mrsh/craham/revue/tabularia/print.php?dossier=dossier11\&file=02paquet.xml 


\begin{abstract}
$A$ : Original perdu.
$B$ : Copie d'après $A, \mathrm{XIII}^{\mathrm{e}}-\mathrm{XIV}^{\mathrm{e}}$ siècles: cartulaire, Bibl. mun. Rouen, ms 1207

[Y 51], fol. 36-v.

$C$ : Copie d'après $B$, fin XVI e siècle: BnF, nouv. acq. lat., ms 2412, ${ }^{\circ} 110$, p. 85.
\end{abstract}

\title{
XXXV
}

1190-1219 - Raoul d'Argences concède à Girard de Vittefleur ${ }^{185}$ une masure située à Veules-les-Roses ${ }^{186}$.

$A$ : Original perdu.

$B$ : Copie d'après $A, \mathrm{XIII}^{\mathrm{e}}-\mathrm{XIV}^{\mathrm{e}}$ siècles: cartulaire, Bibl. mun. Rouen, ms 1207 [Y 51], fol. 37 .

$C$ : Copie d'après $B$, fin $\mathrm{XVI}^{\mathrm{e}}$ siècle: $\mathrm{BnF}$, nouv. acq. lat., $\mathrm{ms} 2412, \mathrm{n}^{\circ} 112$, p. 86-87.

\section{XXXVI}

1190-1219 - Raoul d'Argences concède à Robert Wapaillaria d'Escruteville ${ }^{187}$ un demi-villainage situé à Escruteville.

$A$ : Original perdu.

$B$ : Copie d'après $A, X^{X} I^{e}-{ }^{e} I^{e}{ }^{e}$ siècles: cartulaire, Bibl. mun. Rouen, ms 1207 [Y 51], fol. 37 .

$C$ : Copie d'après $B$, fin $X_{V I}{ }^{e}$ siècle: $B n F$, nouv. acq. lat., ms $2412, n^{\circ} 113$, p. 87.

\section{XXXVII}

1190-1219 - Raoul d'Argences afferme des terres situées à Évecquemont ${ }^{188}$ à douze de ses vassaux.

$A$ : Original perdu.

$B$ : Copie d'après $A, X$ XIII $^{\mathrm{e}}-\mathrm{XIV}^{\mathrm{e}}$ siècles: cartulaire, Bibl. mun. Rouen, ms 1207 [Y 51], fol. 38.

$C$ : Copie d'après $B$, fin XVI ${ }^{e}$ siècle: $B n F$, nouv. acq. lat., ms 2412, $n^{\circ} 116$, p. 89-9o.

\section{XXXVIII}

1190-1219 - Raoul d'Argences confirme à Gauthier de Francheville ${ }^{189}$ un tènement situé à Coutremoulins ${ }^{190}$.

185. Dép. Seine-Maritime, cant. Cany-Barville.

186. Dép. Seine-Maritime, cant. Saint-Valéry-en-Caux. Contrairement à ce qui est indiqué par l’auteur de la copie du XVI ${ }^{e}$ siècle, il ne doit pas s'agir de Veulettes-sur-Mer (dép. Seine-Maritime, cant. Cany-Barville), mais bien de Veules-les-Roses, localité où l'abbaye de Fécamp disposait d'un bourgage (Mabille, 1953, p. 105).

187. Aujourd'hui Écretteville-lès-Baons (dép. Seine-Maritime, cant. Yvetôt).

188. Dép. Yvelines, cant. Meulan.

189. Peut-être: Francheville, dép. Eure, cant. Breteuil.

190. Dép. Seine-Maritime, cant. Valmont. 


\begin{abstract}
$A$ : Original perdu.
$B$ : Copie d'après $A$, XIII $^{\mathrm{e}}-\mathrm{XIV}^{\mathrm{e}}$ siècles: cartulaire, Bibl. mun. Rouen, ms 1207

[Y 51], fol. 38v.

$C$ : Copie d'après $B$, fin XVI ${ }^{e}$ siècle: BnF, nouv. acq. lat., $m s$ 2412, ${ }^{\circ} 118$, p. 91.
\end{abstract}

\title{
XXXIX
}

1190-1219 - Raoul d'Argences concède à Ralbert Radigel, de Caudebec ${ }^{191}$, une masure située à Caudebec.

$A$ : Original perdu.

$B$ : Copie d'après $A, \mathrm{XIII}^{\mathrm{e}}-\mathrm{XIV}^{\mathrm{e}}$ siècles: cartulaire, Bibl. mun. Rouen, ms 1207 [Y 51], fol. 38v, 39 .

$C$ : Copie d'après $B$, fin $\mathrm{XVI}^{\mathrm{e}}$ siècle: $\mathrm{BnF}$, nouv. acq. lat., ms 2412, $\mathrm{n}^{\circ}$ 119, p. 91-92.

XL

119o-1219 - Raoul d'Argences confie l'administration d'une partie de la forêt d'Eawy ${ }^{192}$ à maître Matthieu et à Guillaume, deux clercs.

$A$ : Original perdu.

$B$ : Copie d'après $A$, XIII $^{e}-X{ }^{e}$ siècles: cartulaire, Bibl. mun. Rouen, ms 1207 [Y 51], fol. 39v, 3obis.

$C$ : Copie d'après $B$, fin XVI ${ }^{e}$ siècle: $B n F$, nouv. acq. lat., ms $2412, n^{\circ} 122$, p. 94-95.

\section{XLI}

1190-1219 - Raoul d'Argences concède à Hugues de Paluel ${ }^{193}$, prêtre, une vavassorie qui a appartenu à Lambert, prêtre de Paluel auparavant.

$A$ : Original perdu.

$B$ : Copie d'après $A, X^{X} I^{e}-{ }^{e}{ }^{2}{ }^{e}$ siècles: cartulaire, Bibl. mun. Rouen, ms 1207 [Y 51], fol. 3obis.

$C$ : Copie d'après $B$, fin $\mathrm{XVI}^{\mathrm{e}}$ siècle: $\mathrm{BnF}$, nouv. acq. lat., $\mathrm{ms} 2412, \mathrm{n}^{\circ}$ 123, p. 95-96.

\section{XLII}

1190-1219 - Raoul d'Argences échange avec Eustache de Faillecot une rente contre une masure située aux Dalles ${ }^{194}$.

$A$ : Original perdu.

191. Aujourd'hui Caudebec-en-Caux, dép. Seine-Maritime, chef-lieu de cant.

192. Dép. Seine-Maritime.

193. Dép. Seine-Maritime, cant. Cany-Barville.

194. Probablement aujourd'hui le hameau des Petites-Dalles, partagé entre Sassetot-le-Mauconduit (dép. Seine-Maritime, cant. Valmont) et Saint-Martin-aux-Buneaux (dép. Seine-Maritime, cant. Cany-Barville). 
$B$ : Copie d'après $A, \mathrm{XIII}^{\mathrm{e}}$-XIV $^{\mathrm{e}}$ siècle: cartulaire, Bibl. mun. Rouen, ms 1207 [Y 51], fol. 3obis.

$C$ : Copie d'après $B$, fin XVI e siècle: BnF, nouv. acq. lat., ms 2412, nº 124, p. 96.

\section{XLIII}

119o-1219 - Raoul d'Argences confirme une terre de Contremoulins ${ }^{195}$ à Geoffroi de Contremolins, vassal de l'abbaye.

$A$ : Original perdu.

$B$ : Copie d'après $A, \mathrm{XIII}^{\mathrm{e}}-\mathrm{XIV}^{\mathrm{e}}$ siècle: cartulaire, Bibl. mun. Rouen, ms 1207 [Y 51], fol. 3obisv.

$C$ : Copie d'après $B$, fin XVI ${ }^{e}$ siècle: BnF, nouv. acq. lat., ms 2412, $\mathrm{n}^{\circ} 125$, p. 96-97.

\section{XLIV}

1190-1219 - Raoul d'Argences confirme deux moulins situés à Rye ${ }^{196}$ à Helie, fils de Sugelbert.

A: Original: Arch. dép. Seine-Maritime, 7 H 43.

\section{XLV}

119o-1219 - Aveu de Pierre de Canteleu. Raoul d'Argences et lui-même font part de son hommage à l'abbé. Le vassal confirme les donations faites par son père à l'abbaye, pour le salut de ses ancêtres.

$A$ : Original perdu.

$B$ : Copie d'après $A, \mathrm{XIII}^{\mathrm{e}}-\mathrm{XIV}^{\mathrm{e}}$ siècle: cartulaire, Bibl. mun. Rouen, ms 1207 [Y 51], fol. 6ov.

$C$ : Copie d'après $B$, fin XVI ${ }^{e}$ siècle: BnF, nouv. acq. lat., ms 2412, ${ }^{\circ}$ 210, p. 184.

195. Dép. Seine-Maritime, cant. Valmont. 196. Sussex. 